\title{
Looking for the least luminous BL Lacertae objects
}

\author{
A. Capetti and C. M. Raiteri \\ INAF - Osservatorio Astrofisico di Torino, via Osservatorio 20, 10025 Pino Torinese, Italy \\ e-mail: capetti@oato.inaf.it
}

Received 13 February 2015 / Accepted 22 May 2015

\begin{abstract}
Among active galactic nuclei, BL Lac objects show extreme properties that have been interpreted as the effect of relativistic beaming on the emission from a plasma jet oriented close to the line of sight. The Doppler amplification of the jet emission makes them ideal targets for studying jet physics. In particular, low-power BL Lacs (LPBL) are very interesting because they probe the jet formation and emission processes at the lowest levels of accretion. However, they are difficult to identify since their emission is swamped by the radiation from the host galaxy in most observing bands. In this paper we propose a new LPBL selection method based on the mid-infrared emission, in addition to the traditional optical indices. We considered the radio-selected sample of Best \& Heckman (2012, MNRAS, 421, 1569) and cross-matched it with the WISE all-sky survey. In a new diagnostic plane including the W2-W3 color and the $\operatorname{Dn}(4000)$ index, LPBL are located in a region scarcely populated by other sources. By filtering objects with small emission line equivalent width, we isolated $36 \mathrm{LPBL}$ candidates up to redshift 0.15 . Their radio luminosity at $1.4 \mathrm{GHz} \mathrm{spans}$ the range $\log L_{\mathrm{r}}=39.2-41.5\left[\mathrm{erg} \mathrm{s}^{-1}\right.$ ]. Considering the completeness of our sample, we analyzed the BL Lac luminosity function (RLF), finding a dramatic paucity of LPBL with respect to the extrapolation of the RLF toward low power. This requires a break in the RLF located at $\log L_{\mathrm{r}} \sim 40.6\left[\mathrm{erg} \mathrm{s}^{-1}\right.$ ]. The consequent peak in the BL Lacs number density is possibly the manifestation of a minimum power required to launch a relativistic jet.
\end{abstract}

Key words. galaxies: active - BL Lacertae objects: general - galaxies: jets

\section{Introduction}

BL Lac objects (BL Lacs), together with flat-spectrum radio quasars (FSRQs), form a class of active galactic nuclei (AGNs) known as blazars. Like the other radio-loud AGNs, they are thought to be powered by a central supermassive black hole, surrounded by an accretion disk. Two plasma jets are ejected in the direction orthogonal to the disk. Among radio-loud AGNs, blazars show extreme properties, such as strong flux variability on the whole electromagnetic spectrum even on intra-day time scales (Wagner et al. 1996), variable optical and radio polarization (Aller et al. 1996; Smith 1996), and apparent superluminal motion of radio components (Kellermann et al. 2004). The explanation of this observing evidence requires that one of the relativistic plasma jets be closely aligned with the line of sight, causing Doppler beaming of the jet emission (Urry \& Padovani 1995). This makes blazars the ideal candidates for studying the physics of AGN jets. In particular, the least luminous blazars are extremely interesting because they can probe the jet formation and emission processes at the lowest levels of accretion.

The classical distinction between BL Lacs and FSRQs is based on the strength of the emission lines in the optical spectrum, with BL Lacs being identified by rest-frame equivalent widths (EW) less than $5 \AA$ (Stickel et al. 1991). The blazar spectral energy distribution (SED) is dominated by the nonthermal emission from the jet. It shows two bumps: the lowenergy one (from radio to UV-X-rays) is due to synchrotron radiation, while the high-energy bump (X-and $\gamma$-rays) is probably due to an inverse-Compton process off the same relativistic electrons that produce the synchrotron photons (Konigl 1981). Depending on the frequency of the synchrotron peak $v_{\mathrm{p}}$ in the SED, BL Lacs have been divided into low-, intermediate-, and high-energy synchrotron peaked BL Lacs. The objects of the first class (named LBL or LSP) have $v_{\mathrm{p}}<10^{14} \mathrm{~Hz}$. For those of the second class (IBL or ISP) $10^{14}<v_{\mathrm{p}}<10^{15} \mathrm{~Hz}$, and for objects of the third class (HBL or HSP), $v_{\mathrm{p}}>10^{15} \mathrm{~Hz}$ (Abdo et al. 2010). It has been claimed that blazar SEDs follow a blazar sequence (Fossati et al. 1998; Donato et al. 2001), linking the SEDs of FSRQs at the highest synchrotron peak luminosities $L_{\mathrm{p}}$ and lowest $v_{\mathrm{p}}$ to the SEDs of LBL, IBL, and finally HBL, when progressively decreasing $L_{\mathrm{p}}$ while increasing $v_{\mathrm{p}}$. This view has been criticized, for example, by Giommi et al. (2012a), who underline the selection effects of flux-limited surveys and lack of redshift determination for the likely high- $L_{\mathrm{p}}-$ high- $\nu_{\mathrm{p}}$ sources.

The difficulty of finding low-luminosity BL Lacs comes from the dilution of the jet component by the host-galaxy emission in the optical and near-IR bands, as well as from the flux limit of the available surveys. As a result, their radio luminosity function (RLF) is only determined above $\log L_{\mathrm{r}} \sim 40.5\left[\mathrm{erg} \mathrm{s}^{-1}\right]$ (e.g., Wolter et al. 1994; Padovani et al. 2007). The analysis of Marchã \& Caccianiga (2013) reaches lower luminosities when also including Type 0 and weak-lined radio galaxies. Our aim is to find a substantial number of low-power BL Lacs (LPBL) and to build their RLF down to a poorly studied regime of radio power.

This paper is organized as follows. In Sect. 2 we present a new selection method for identifying BL Lacs that involves both optical and mid-infrared (MIR) properties. This leads to singling out 36 BL Lac candidates with redshift $z \leq 0.15$ and radio luminosities in the range $\log L_{\mathrm{r}} \sim 39$ to $42\left[\mathrm{erg} \mathrm{s}^{-1}\right.$ ] in Sect. 3. The various sources of incompleteness in our sample are considered in Sects. 4 and 5, while the resulting BL Lac RLF is derived and also discussed in the framework of the AGN unification scheme in Sect. 6. After a summary, our conclusions are drawn in Sect. 7. 

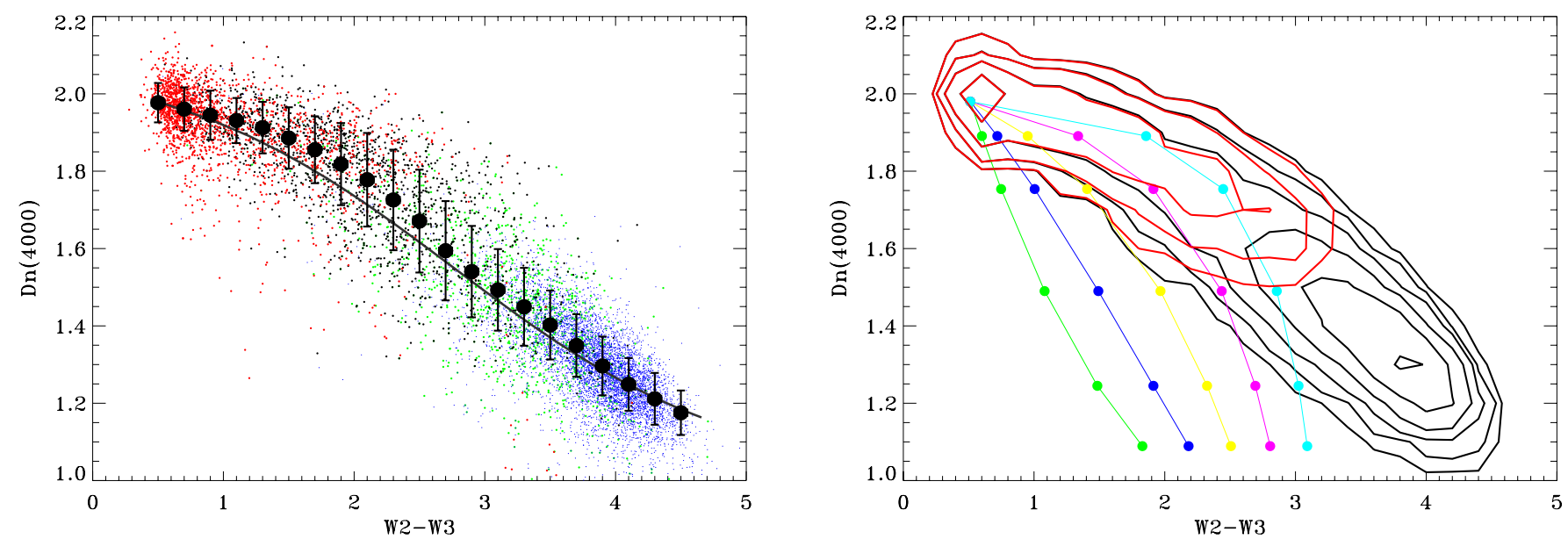

Fig. 1. Left: relation between the Dn(4000) index and the W2-W3 color measured from the sample of 21065 bright nearby galaxies (see text for the selection criteria). For each bin in W2-W3, the large black dots denote the mean value and dispersion of the best Gaussian fit to the Dn(4000) distributions. Each spectroscopic class is highlighted with a color: red for lineless galaxies, black for LINERs, green for Seyferts, and blue for star-forming galaxies. The solid line represents the result of a toy model where a fixed fraction of a blue light component of increasing strength is reprocessed into MIR radiation. Right: simulated Dn(4000) versus W2-W3 tracks obtained by adding a power-law component of increasing strength with a spectral index increasing from 0 (green) to 1 (cyan) with a step of 0.25 to the SED of a quiescent galaxy. Within each track we label the ratios of $0,0.1,0.3,1,3$, and 10 between the jet and galactic components. The black lines trace the iso-density contours of the distribution shown in the left panel; the red lines show the iso-densities contours for the objects with all lines rest frame $E W<5 \AA$.

Throughout the paper we adopt a cosmology with $H_{0}=$ $70 \mathrm{~km} \mathrm{~s}^{-1} \mathrm{Mpc}^{-1}, \Omega_{\mathrm{M}}=0.29$, and $\Omega_{\Lambda}=0.71$. When we speak of radio luminosities $L_{\mathrm{r}}$, we mean $v_{\mathrm{r}} l_{\mathrm{r}}$, where the monochromatic luminosity $l_{\mathrm{r}}$ is measured at $\nu_{\mathrm{r}}=1.4 \mathrm{GHz}$. Moreover, we distinguish between radio luminosities in cgs units, $L_{\mathrm{r}}$, and those in MKS units, $P_{\mathrm{r}}$.

\section{The selection method}

As mentioned, the peculiarity of BL Lacs is their non-thermal emission from a relativistic jet seen at a small angle with respect to the line of sight. As a consequence, their optical spectrum is either featureless or only presents emission lines of small EW. This is because lines (when present) are isotropic, while the jet emission is highly beamed. Similarly, the jet dilutes the stellar absorption features. BL Lacs have been historically selected by setting a limit of $5 \AA$ to the rest-frame EW of any emission line (Stickel et al. 1991). Alternatively, they have been recognized as objects with low Dn(4000) index ${ }^{1}$. The Dn(4000) limit was set to 1.33 by Stocke et al. (1991), then later relaxed to 1.67 by Marcha et al. (1996) and Plotkin et al. $(2010)^{2}$. Nevertheless, passive elliptical galaxies have $\operatorname{Dn}(4000) \sim 2$ (see below), so that we can expect that the $\operatorname{Dn}(4000)$ constraint can be further relaxed to find LPBL (see also Marchã \& Caccianiga 2013).

We propose to loosen the $\operatorname{Dn}(4000)$ limit but to include a further selection tool based on the MIR emission. In fact, in active galaxies the MIR is also an isotropic quantity, owing to the reprocessing of (mainly) UV photons from the interstellar dust, while in BL Lacs it also receives a non-thermal contribution from the jet that alters the overall SED shape. As we discuss in detail below, the resulting MIR properties enable us to separate BL Lacs from other classes of active galaxies.

\footnotetext{
1 Defined according to Balogh et al. (1999) as the ratio between the flux density measured on the red side of the Ca II break (4000-4100 A) and that on the blue side (3850-3950 ̊).

2 Various authors use different definitions of the Ca II break strength. Our parameterization is related to those found in the literature as $\operatorname{Dn}(4000)=1 /(1-C)($ Landt et al. 2002).
}

The WISE satellite provides us with an all-sky survey in the four bands $\mathrm{W} 1, \mathrm{~W} 2, \mathrm{~W} 3$, and W4, which are centered at 3.4, 4.6, 12, and $22 \mu \mathrm{m}$, respectively (Wright et al. 2010). The best-suited color for our purposes is obtained from the W2 and W3 bands, because it has high wavelength leverage and level of completeness.

Before we can proceed to use the MIR observations for the BL Lacs selection, we must explore the relationship between the optical and MIR properties of galaxies in general. For this purpose, we consider the 927552 SDSS DR 7 spectra of the 818333 galaxies (MPA-JHU sample hereafter) in the valueadded spectroscopic catalog 3 produced by the group from the Max Planck Institute for Astrophysics, and The Johns Hopkins University (Brinchmann et al. 2004; Tremonti et al. 2004).

Our aim is to detect the faintest BL Lacs, so we started to analyze galaxies up to $z=0.1$ and considered only massive objects, since they are the typical BL Lac hosts. We then filtered objects with high-quality data with the following criteria:

i) redshift $z \leq 0.1$,

ii) error on $\operatorname{Dn}(4000)<0.02$,

iii) signal-to-noise $(\mathrm{S} / \mathrm{N})$ in both $\mathrm{W} 2$ and $\mathrm{W} 3$ bands $>10$, iv) stellar velocity dispersion $>100 \mathrm{~km} \mathrm{~s}^{-1}$.

In Fig. 1 (left panel) we compare the W2-W3 color with the Dn(4000) index for the 21065 selected galaxies. The presence of a clear sequence emerges, with the Dn(4000) value decreasing with increasing W2-W3. In each W2-W3 bin, we estimated the mean value and dispersion of the best Gaussian fit to the Dn(4000) distribution.

Taking advantage of the SDSS spectra, we can consider the various classes of galaxies separately. They are located in different areas of this diagram: the passive galaxies are clustered at the top left, with $\mathrm{Dn}(4000) \sim 2$ and W2-W3 $\sim 0.5$. Proceeding toward the opposite corner of the diagram we find LINERs, most of them having Dn(4000) 1.5-2.0 and $1<\mathrm{W} 2-\mathrm{W} 3<3$, and then Seyfert galaxies with $\mathrm{Dn}(4000) \sim 1.5$ and $3<\mathrm{W} 2-\mathrm{W} 3<4$. In the bottom right hand corner, we find star-forming galaxies,

\footnotetext{
3 Available at http://www . mpa-garching.mpg.de/SDSS/
} 

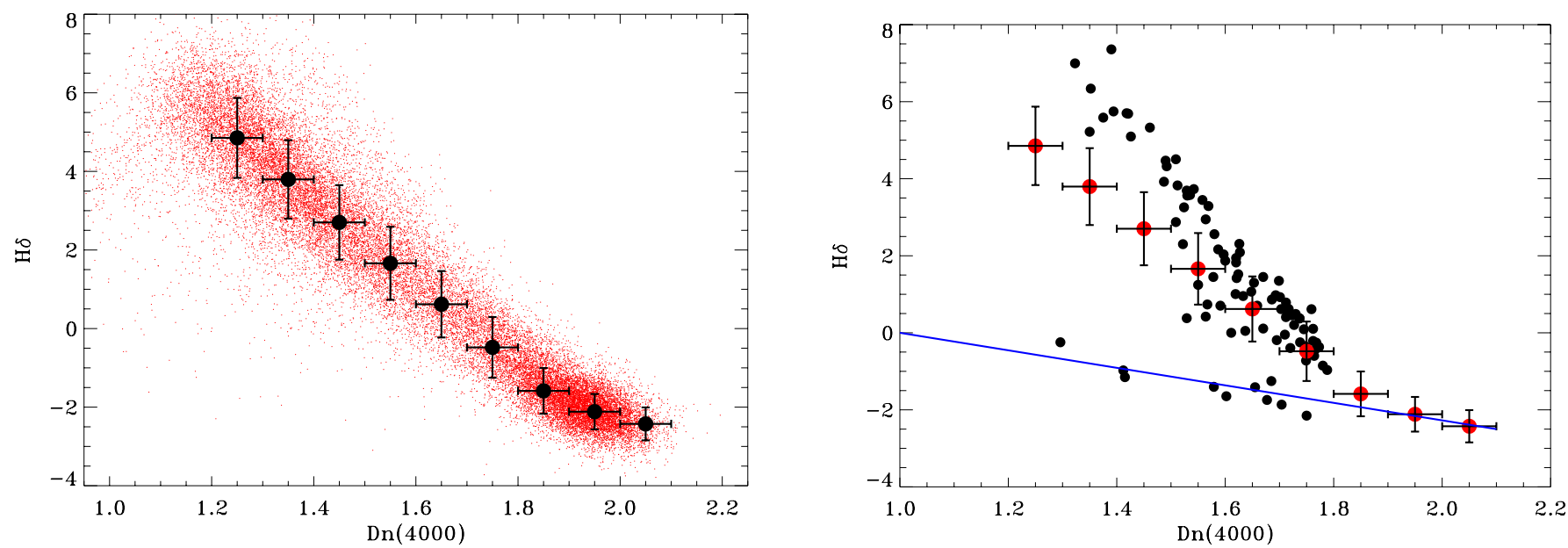

Fig. 2. Left: relation between the Dn(4000) and $\mathrm{H} \delta$ indices from the SDSS subsample of 21065 galaxies. For each bin in Dn(4000), the black dots indicate the mean value and dispersion of the best Gaussian fit to the $\mathrm{H} \delta$ distribution. Right: $\mathrm{H} \delta$ versus $\mathrm{Dn}(4000)$ for the outliers of the Dn(4000) versus W2-W3 relation (black dots). The red dots label, for each bin in Dn(4000), the mean value and dispersion of the best Gaussian fit to the $\mathrm{H} \delta$ distribution for the overall SDSS galaxies population. The straight blue line is the expected locus of BL Lacs, obtained by varying the ratio between jet and galactic emission (see text for details).

with low Dn(4000) values $(\sim 1.2-1.3)$ and large excesses in the W3 band. Therefore, all spectroscopic classes of galaxies follow the same overall sequence possibly because, regardless of the origin of the blue/UV light, the same mechanism of reprocessing into the MIR is operating.

To support this interpretation further we built a very simple model. We added a growing contribution of blue light to the SED of a passive galaxy and adopted a constant reprocessing efficiency into MIR emission. The track obtained is shown in Fig. 1 (left panel) and follows the central values of the distributions remarkably well.

It is important to note that the same sequence is followed by the objects with low values of the emission lines EW, the sources among which we expect, in principle, to find BL Lacs. By setting a limit to the lines rest frame of $E W<5 \AA$, we obtain the red contours overplotted in Fig. 1. The distribution of this subsample follows that of the general galaxies population closely up to $\mathrm{W} 2-\mathrm{W} 3 \sim 3$. The low EW objects only avoid the region of largest MIR colors, which are typical of star-forming galaxies.

We can now explore the expected behavior of BL Lac objects in the same diagram. This was simulated by adding to the SED of a quiescent galaxy a jet component $F_{\text {jet }}$. For the quiescent galaxy we use a $\operatorname{Dn}(4000)=1.98$ and $\mathrm{W} 2-\mathrm{W} 3=0.5$, i.e. the coordinates of the upper left average point in Fig. 1, while for the jet component we adopt a power-law spectral shape in the MIR-optical frequency range of the form $F_{\text {jet }}=F_{0} v^{-\alpha}$. When $F_{\text {jet }}$ increases, the W2-W3 color grows, owing to the larger relative contribution of $F_{\text {jet }}$ in the W3 band than in the W2 band with respect to the galactic emission. At the same time, an excess of blue light emerges, thus reducing the Dn(4000) index (see Fig. 1, right panel). Depending on the value of $\alpha$, the expected locus of BL Lacs varies. The curves obtained with different values of $\alpha$ can be overplotted onto the relation between $\mathrm{W} 2-\mathrm{W} 3$ and Dn(4000) obtained above. All the simulated tracks are steeper than the phenomenological trend traced by the sample considered above. As a result, the expected BL Lac tracks exit the region where the SDSS galaxies are located. The transition into the forbidden zone occurs at $\mathrm{Dn}(4000) \sim 1.8$ for the flatter BL Lac SED and at lower values for softer SEDs. The visual inspection of broad-band SEDs of various classes of BL Lacs (e.g., Giommi et al. 2012b; Raiteri et al. 2014) indicates that an optical-to-MIR index $\alpha \sim 0.5$ is typical of HBL, while $\alpha \sim 1$ can be associated to LBL.

In other words, the presence of a BL Lac nucleus produces a substantial excess of blue light that corresponds to a smaller MIR excess than in the case of active or star-forming galaxies. We conclude that this diagnostic plane can be used to isolate BL Lac objects.

\subsection{Outliers in the Dn(4000) versus W2-W3 plane}

Although SDSS galaxies define a clear locus in the Dn(4000) versus W2-W3 plane, several outliers are present in the region where we expect to find BL Lacs. These objects thus deserve further investigation in order to ensure the purity of the sample. We then individually consider the 112 objects (representing only $0.5 \%$ of the 21065 selected galaxies) lying below the average $\mathrm{Dn}(4000)$ versus $\mathrm{W} 2-\mathrm{W} 3$ relation by more than $3 \sigma$. In nine cases a bright star is located close to the galaxy's center and is covered by the SDSS aperture; this causes the reduced Dn(4000) value. In 11 objects we are dealing with clear misidentifications, since they are all high-redshift QSOs $(z=1.5-2.3)$.

There are still 92 objects. When looking at their SDSS spectra, it emerges that most of them have a pronounced absorption corresponding to the Balmer lines. These objects are often referred to as E+A galaxies (Goto et al. 2003). The presence of the A stars produces a substantial contribution in the $4000 \AA$ spectral region, leading to low $\mathrm{Dn}(4000)$ values. The lack of the corresponding increase in the WISE color is due to the spectral shape of the A stars, with a sharp drop below the Balmer break. The paucity of UV photons, which are most effectively absorbed by dust and are consequently the dominant mechanism of dust heating, accounts for the reduced MIR emission. Nonetheless, such sources can be easily recognized by measuring their $\mathrm{H} \delta$ index (Worthey \& Ottaviani 1997).

In Fig. 2 (left panel) we show the relation between the $\mathrm{Dn}(4000)$ and $\mathrm{H} \delta$ indices for the same sample of SDSS galaxies as used above. The $\mathrm{H} \delta$ index monotonously decreases with increasing Dn(4000).

In Fig. 2 (right panel), we plot the 92 objects located in the forbidden zone in Fig. 1 in the same diagram. They clearly separate into two sequences. The first follow the general $\mathrm{H} \delta$ versus 


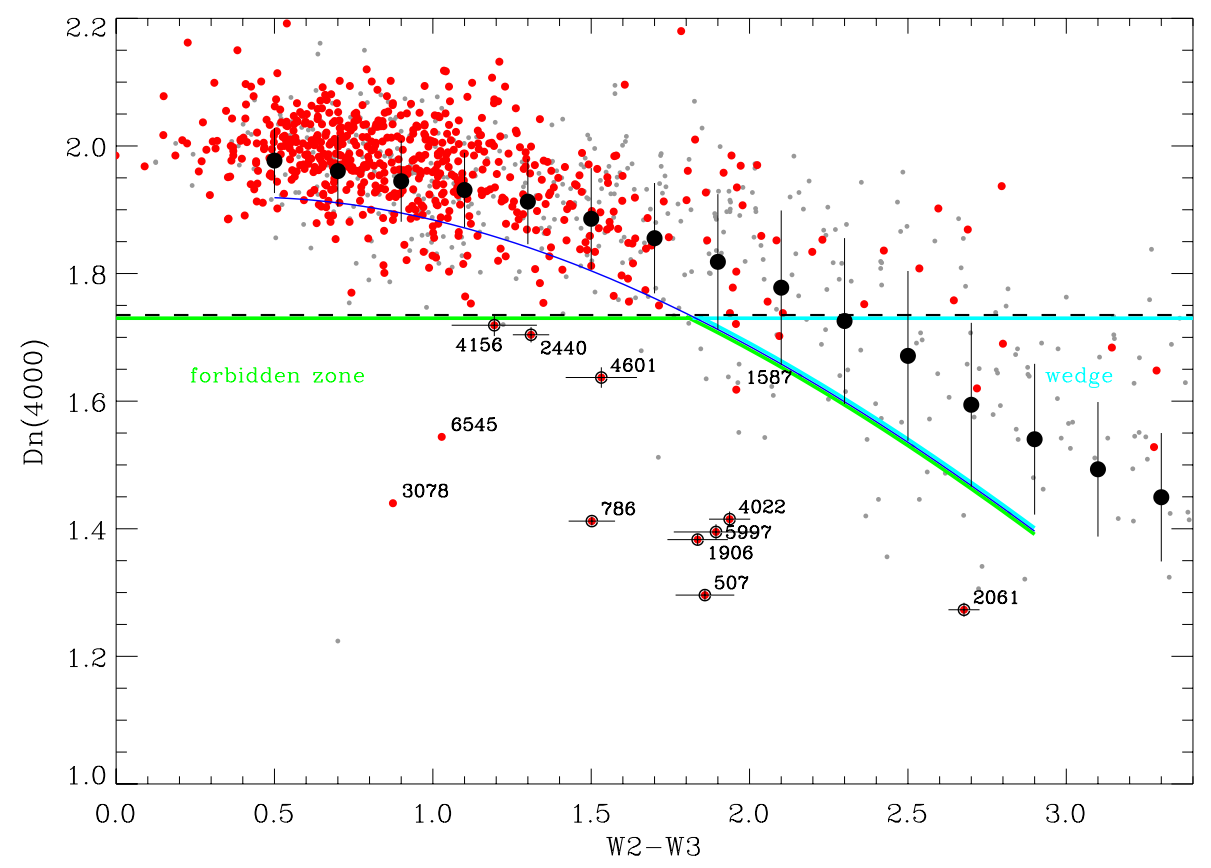

Fig. 3. Dn(4000) versus W2-W3 diagram. Black filled circles represent the relationship derived for the MPA-JHU sample that is shown in Fig. 1. The blue line is a polynomial fit to the $1 \sigma$ limit of this relationship. Gray dots represent the sources in the BH12-A sample with $z \leq 0.1$ and $S / N>2$ in the W2 and W3 bands. Red dots highlight the objects with rest-frame emission line $E W<5 \AA$ and a FIRST central counterpart. Red dots circled in black are the nine BL Lacs candidates, i.e. those sources with $\mathrm{Dn}(4000)<1.735$ (shown by the horizontal dashed line) that meet the additional constraint on the $\mathrm{H} \delta$ index (see Fig. 4). The boundaries of the forbidden zone and wedge region are highlighted with a green and a cyan line, respectively.
Dn(4000) trend, and they are most likely E+A galaxies. Their position within the sequence depends on the fractional contribution of A stars in each given object. The second group of objects is instead located where BL Lacs are expected. This region connects the bottom right hand corner of the diagram, which is characteristic of quiescent galaxies, to the location of a featureless source, having $\operatorname{Dn}(4000)=1$ and $\mathrm{H} \delta=0$. Indeed, five out of ten of them are associated with a radio source with a flux density higher than $\sim 50 \mathrm{mJy}$, which is a clear indication for the presence of non-thermal emission. We recover them as BL Lac candidates in Sect. 3.

Summarizing, the proposed tool for isolating BL Lacs from a large population of galaxies based on the Dn(4000) versus WISE W2-W3 color diagram appears to be promising, particularly for HBL objects, which are expected to populate a forbidden zone in this diagram. However, the presence of outliers in this region requires

i) visually exploring the SDSS data to remove objects whose spectrum is contaminated by a nearby star,

ii) individually checking for misidentified objects,

iii) most importantly, including a further diagram formed by the $\mathrm{Dn}(4000)$ and $\mathrm{H} \delta$ indices in the analysis in order to exclude $\mathrm{E}+\mathrm{A}$ galaxies.

After such an analysis we are left with only five possible contaminants to the BL Lacs population, i.e. five galaxies (out of 21065 ) with a low value of $\operatorname{Dn}(4000)$ but lack both MIR excess and radio emission. The nature of these objects is unclear; however, due to their extreme rarity, we do not expect them to significantly affect the results of our study.

\section{Looking for the least luminous BL Lacs}

We looked for the least luminous BL Lacs by considering the sample of 18286 radio sources built by Best \& Heckman (2012, hereafter BH12). They combined the MPA-JHU sample with the NVSS and the FIRST surveys, the cross-matching going down to a flux density level of $5 \mathrm{mJy}$ in the NVSS. We first focused on the 7302 objects they classified as radio-AGNs, separating them from the star-forming galaxies. These authors also restricted their work to radio sources within the main galaxy sample (Strauss et al. 2002), comprising those galaxies with magnitudes in the range $14.5<r<17.77$ and redshift range $0.01<z<0.3$. The 7302 sources (hereafter the BH12-A sample) were cross-matched with the AllWISE catalog ${ }^{4}$ using a 3 arcsec search radius and finding 7242 counterparts ${ }^{5}$.

Since our aim is to detect the faintest BL Lacs, we started to analyze the closest Universe, up to $z=0.1$. In Fig. 3 we present the Dn(4000) versus W2-W3 plot for all objects with $z \leq 0.1$ and with S/N of WISE data in the W2 and W3 bands $>2$. A comparison with Fig. 1 shows that most radio galaxies are located in the same area as the MPA-JHU sample and that we are now dealing with mostly weakly active galaxies, with $\operatorname{Dn}(4000) \sim 2$ and W2-W3 in the 0.5-1.5 range, while the locus of star-forming galaxies is poorly populated. When further selecting objects with i) rest-frame EW of the main emission lines $<5 \AA$ and ii) existence of a FIRST central counterpart to exclude double sources lacking a radio core, we are left with 609 sources (Fig. 3), only a few of which lying in the forbidden zone.

We searched for BL Lac candidates among those selected objects whose ratio between the jet to galactic emission at $3900 \AA$ is $f>1 / 3$, corresponding to $\mathrm{Dn}=\left(\mathrm{Dn}^{0}+f\right) /(1+f)<1.735$, having adopted a $\mathrm{Dn}(4000)$ index for quiescent galaxies $\mathrm{Dn}^{0}=$ 1.98 (see the previous section $)^{6}$. The choice of the $f$ lower limit is arbitrary, since it is a compromise between the possibility of detecting a non-thermal signature (hence distinguishing a BL Lac candidate from a passive galaxy) and the requirement of completeness. However, this assumption will be included selfconsistently in the analysis presented in the following sections. In Fig. 3 there are 12 objects with $\mathrm{Dn}(4000)<\mathrm{Dn}_{\max }=1.735$ and at the same time below the $1 \sigma$ limit traced by the MPA-JHU sample (see Sect. 2). These are labeled with an ID number.

\footnotetext{
4 Available at http://irsa.ipac.caltech.edu/

5 Actually 7271, but 29 are double identifications of which we kept only the closest object.

$6 \operatorname{Dn}(4000)=1.735$ corresponds to $C=0.423$.
} 
A. Capetti and C. M. Raiteri: Looking for the least luminous BL Lacertae objects

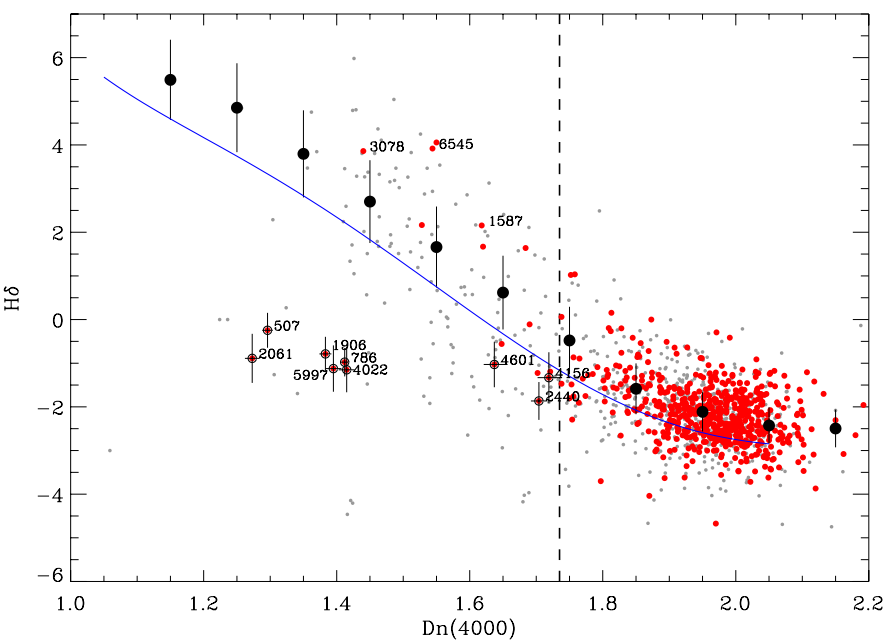

Fig. 4. $\mathrm{H} \delta$ index versus $\mathrm{Dn}(4000)$ diagram. Black filled circles represent the relationship derived for the MPA-JHU sample that is shown in Fig. 2. The blue line is a polynomial fit to the $1 \sigma$ limit of this relationship. All objects above this line are considered E+A contaminants. Gray dots refer to the sources in the BH12-A sample with $z \leq 0.1$ and $S / N>2$ in the W2 and W3 bands. Red dots highlight the objects with the emission line $E W<5 \AA$ and a FIRST central counterpart. Red dots circled in black are the nine BL Lacs candidates. The vertical dashed line indicates the limit $\mathrm{Dn}_{\max }=1.735$.

Figure 4 shows the $\mathrm{H} \delta$ versus $\mathrm{Dn}(4000)$ diagram. If we require that $\mathrm{H} \delta$ is lower than the $1 \sigma$ limit traced by the MPA-JHU sample to exclude contaminating E+A galaxies (post-starburst) as explained in Sect. 2, we are left with nine sources. Their properties are listed in Table 1. Six of them are known ${ }^{7}$ BL Lacs with $\operatorname{Dn}(4000) \sim 1.3-1.4$, while the other three are new candidates with $\mathrm{Dn}(4000)$ values of about 1.7 .

As an example in Fig. 5 we show a SED and SDSS spectrum of one of the selected objects, namely the BL Lac candidate ID $=2440$ at $z=0.076$. The departure of the SDSS spectrum and WISE data from the properly normalized galactic templates highlights the reduced Dn(4000) value of the source, as well as the W3 (and W4) excess, we interpret as due to the presence of the jet emission.

\subsection{Expanding the search to $0.1<z \leq 0.15$}

The BL Lac candidates we found in the $z \leq 0.1$ range have all radio flux densities greater than $18 \mathrm{mJy}$, which is well above the limit of the BH12 sample, 5 mJy. This means that we can detect similar objects at slightly higher redshifts. As a result, to improve the statistical significance of our work, we expand our search for BL Lac candidates to a three times larger space volume, i.e. to $z=0.15$. We follow the same method as presented in the previous section, but we recalibrate all relationships with objects in the proper redshift range (see Fig. 6).

In the redshift range $0.1<z \leq 0.15$, the $\mathrm{Dn}(4000)$ limit that corresponds to $f=1 / 3$ is 1.713 , since the average $\operatorname{Dn}(4000)$ value of quiescent galaxies in this redshift bin is reduced to 1.95. Our selection criteria identify 742 objects with weak emission lines. The Dn(4000) versus W2-W3 diagram (Fig. 7) is very similar to the one obtained at lower redshift, but there are more sources with weak lines in the forbidden zone. In particular, there

\footnotetext{
They are included in the catalog of blazars Roma-BZCAT (Massaro et al. 2009), available at http://www . asdc . asi.it/bzcat/
}
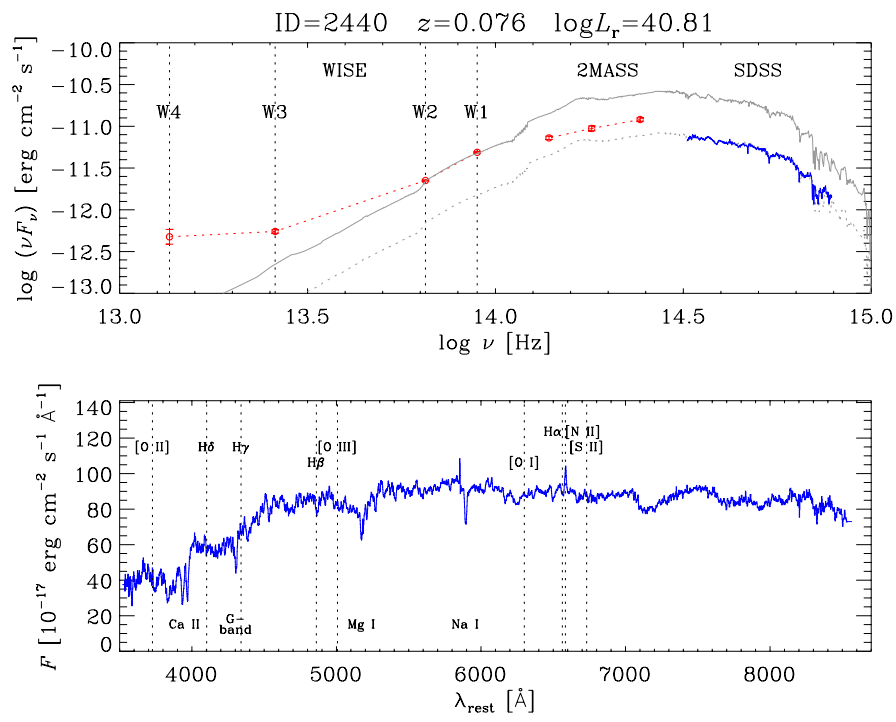

Fig. 5. Top: SED of a new BL Lac candidate, ID $=2440$. Red circles display WISE and dereddened 2MASS flux densities. The SDSS dereddened spectrum is shown in blue. Gray lines represent two SWIRE templates of a 13 Gyr old elliptical galaxy normalized to the W2 band (solid) or to the SDSS spectrum (dotted). The departure of the SDSS spectrum from the latter template highlights the reduced Dn(4000) value of the source, while the W3 (and W4) excess appears from the comparison of the WISE data with the first template. Bottom: rest-frame SDSS spectrum with indication of the main AGN emission lines (upper labels) and galactic absorption lines (lower labels).

are ten objects with low Dn(4000), below 1.4, and well separated from the other sources. The same objects stand alone in the $\mathrm{H} \delta$ versus Dn(4000) plot shown in Fig. 8.

Moreover, there are 16 other objects that are below the $1 \sigma$ limit of the mean Dn(4000) versus W2-W3 relationship. Twelve of them are also below the $1 \sigma$ limit of the mean $\mathrm{H} \delta$ versus Dn(4000) relationship. The SDSS image of the source with ID 3959 shows a contaminating star, so we discard it. The remaining 11 sources are BL Lac candidates.

The properties of all candidates are reported in Table 1.

\subsection{Completing the analysis of the BH12 sample}

So far we only considered the 7302 objects included in the $\mathrm{BH} 12-\mathrm{A}$ radio-AGN sample, i.e. sources from the main galaxy sample where we excluded star-forming galaxies. We checked whether some BL Lacs could hide among the remaining BH12 10984 objects (hereafter BH12-B sample), 10747 of which have a WISE counterpart. If we consider all the sources with $z \leq 0.15$ that meet the selection criteria discussed above, we are left with 377 objects. These reduce to 13 if we require that the position in the Dn(4000) versus W2-W3 is below the average relation defined for the MPA-JHU sample. However, one source is contaminated by a close star, two objects are QSO with mismatched redshift, one has an extremely uncertain redshift. Figure 9 shows the Dn(4000) versus W2-W3 diagram for the BH12-B sample. The remaining nine sources are indicated. When considering that three of them (ID 2309, 8058, 8345) have high $\mathrm{H} \delta$ index and are thus likely E+A galaxy contaminants, we are left with six BL Lac candidates. They are listed in Table 1.

We stress that the constraint based on the W2-W3 color allowed us to remove 54 objects with rest-frame EW of the main emission lines $<5 \AA$ that would instead be selected as 

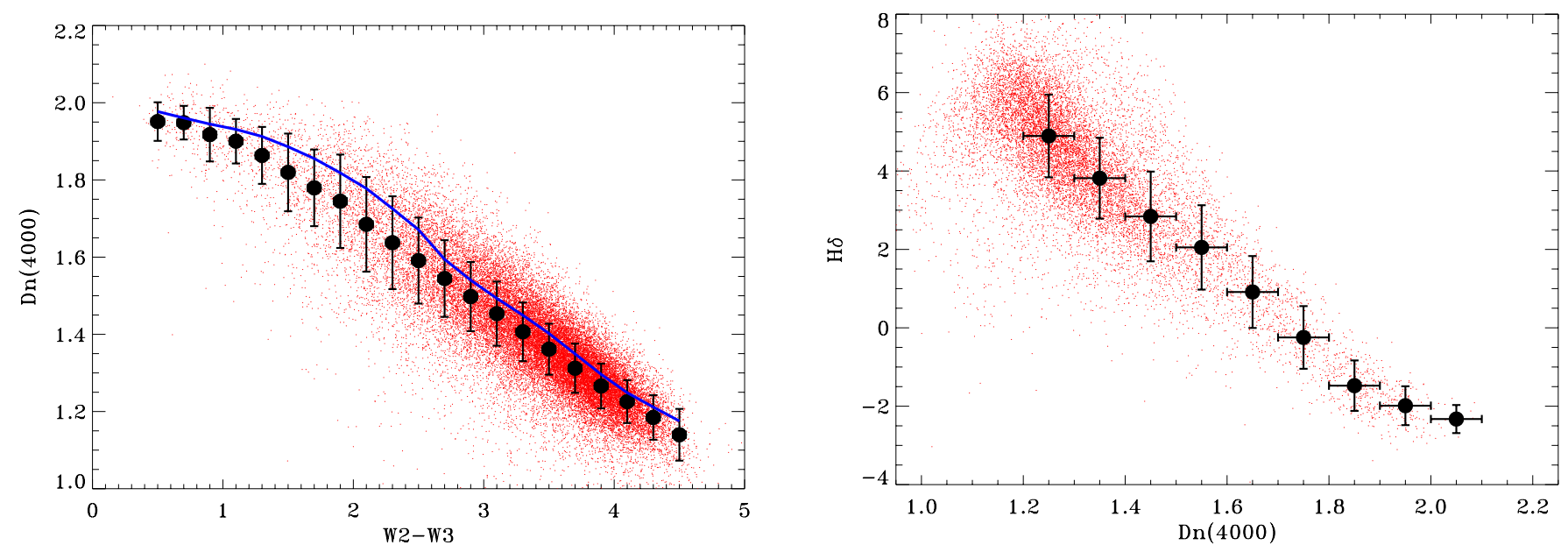

Fig. 6. Same as Fig. 1 (left panel) and Fig. 2 (left panel), but for $0.1<z \leq 0.15$.

Table 1. BL Lac objects and candidates in the BH12 sample at $z \leq 0.15$.

\begin{tabular}{|c|c|c|c|c|c|c|}
\hline ID & RA & Dec & $z$ & $F_{1.4 \mathrm{GHz}}$ & $\log L_{\text {radio }}$ & BZCAT \\
\hline \multicolumn{7}{|c|}{ BH12-A sample with $z \leq 0.1$} \\
\hline 507 & 118.654457 & 39.17994 & 0.096 & 48.80 & 40.20 & BZBJ0754+3910 \\
\hline 2061 & 119.695808 & 27.08766 & 0.099 & 63.50 & 40.34 & BZBJ0758+2705 \\
\hline 1906 & 122.412010 & 34.92701 & 0.082 & 154.9 & 40.56 & BZBJ0809+3455 \\
\hline 5997 & 127.270111 & 17.90440 & 0.089 & 231.8 & 40.81 & BZBJ0829+1754 \\
\hline 786 & 193.445877 & 3.44177 & 0.066 & 78.50 & 40.06 & BZBJ1253+0326 \\
\hline 4156 & 196.580185 & 11.22771 & 0.086 & 117.9 & 40.48 & - \\
\hline 4601 & 199.005905 & 8.58712 & 0.051 & 18.1 & 39.19 & - \\
\hline 2440 & 212.955994 & 52.81670 & 0.076 & 321.6 & 40.81 & - \\
\hline 4022 & 233.009293 & 30.27470 & 0.065 & 61.20 & 39.95 & BZBJ1532+3016 \\
\hline \multicolumn{7}{|c|}{ BH12-A sample with $0.1<z \leq 0.15$} \\
\hline 1315 & 14.083661 & -9.60826 & 0.103 & 99.3 & 40.58 & BZBJ0056-0936 \\
\hline 1805 & 119.060333 & 27.40993 & 0.140 & 20.2 & .17 & - \\
\hline 1903 & 121.805740 & 34.49784 & 0.139 & 32.2 & 40.36 & - \\
\hline 2083 & 132.650848 & 34.92296 & 0.145 & 31.2 & 0.39 & BZBJ0850+3455 \\
\hline 2281 & 144.188004 & 5.15749 & 0.131 & 30.3 & 40.29 & BZUJ0936+0509 \\
\hline 2341 & 151.793533 & 50.39902 & 0.133 & 30.6 & 40.30 & BZBJ $1007+5023$ \\
\hline 6152 & 162.411652 & 27.70362 & 0.144 & 15.6 & 40.08 & - \\
\hline 1845 & 163.433868 & 49.49889 & 0.140 & 56.2 & 40.62 & BZBJ1053+4929 \\
\hline 6428 & 169.276047 & 20.23538 & 0.138 & 117.5 & 40.92 & BZBJ1117+2014 \\
\hline 3591 & 172.926163 & 47.00241 & 0.126 & 127.4 & 40.87 & - \\
\hline 3951 & 183.795746 & 7.53463 & 0.136 & 81.7 & 40.75 & BZBJ1215+0732 \\
\hline 3958 & 185.383606 & 8.36228 & 0.132 & 150.1 & 40.98 & BZBJ1221+0821 \\
\hline 6943 & 194.383087 & 24.21118 & 0.140 & 10.6 & 39.89 & BZBJ1257+2412 \\
\hline 1768 & 201.445267 & 5.41502 & 0.135 & 16.6 & 40.05 & - \\
\hline 7186 & 211.548355 & 22.31630 & 0.128 & 8.3 & 9.70 & - \\
\hline 6982 & 212.616913 & 14.64450 & 0.144 & 434.4 & 41.53 & - \\
\hline 3091 & 216.876160 & 54.15659 & 0.106 & 28.5 & 40.06 & BZBJ1427+5409 \\
\hline 3403 & 217.135864 & 42.67252 & 0.129 & 43.6 & 40.43 & BZBJ1428+4240 \\
\hline 7223 & 223.784271 & 19.33760 & 0.115 & 11.2 & 39.73 & - \\
\hline 4756 & 229.690521 & 6.23225 & 0.102 & 210.9 & 40.89 & - \\
\hline 1087 & 234.945602 & 3.47208 & 0.131 & 11.3 & 39.86 & - \\
\hline \multicolumn{7}{|c|}{ BH12-B sample with $z \leq 0.15$} \\
\hline 1103 & 164.657227 & 56.469769 & 0.143 & 221.4 & 41.23 & BZBJ1058+5628 \\
\hline 1936 & 176.270935 & 19.606350 & 0.022 & 545.4 & 39.91 & - \\
\hline 5076 & 180.764603 & 60.521980 & 0.065 & 171.4 & 40.39 & BZBJ1203+6031 \\
\hline 9591 & 184.054611 & 49.380711 & 0.146 & 9.5 & 39.88 & - \\
\hline 9640 & 227.671326 & 33.584648 & 0.114 & 4.5 & 39.33 & BZBJ1510+3335 \\
\hline 10537 & 233.696716 & 37.265148 & 0.143 & 22.1 & 40.23 & BZBJ1534+3715 \\
\hline
\end{tabular}

Notes. Column description: 1) ID number; 2) RA [deg]; 3) Dec [deg]; 4) redshift; 5) flux density at 1.4 GHz of the FIRST central component [mJy]; 6) the corresponding luminosity [erg s${ }^{-1}$ ] having adopted a null spectral index for the $k$-correction; 7) BZCAT name. 
A. Capetti and C. M. Raiteri: Looking for the least luminous BL Lacertae objects

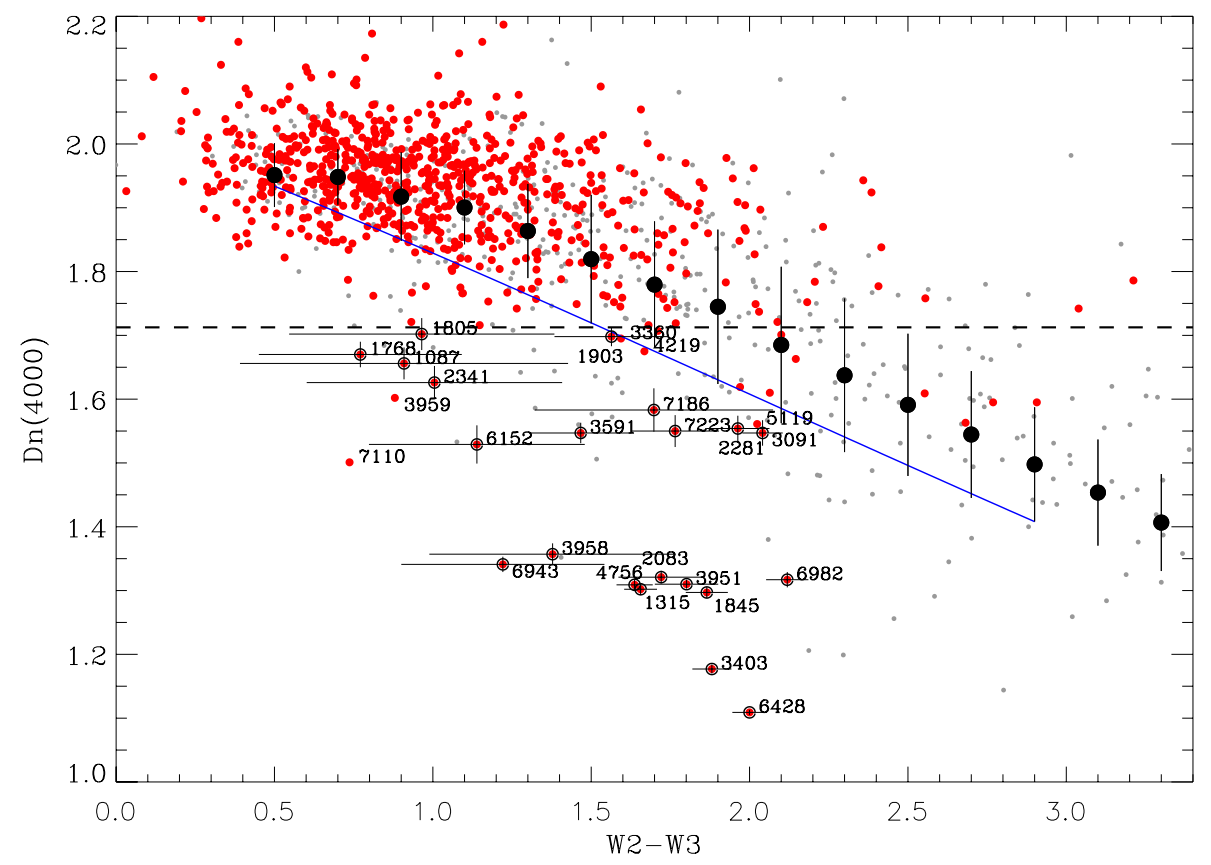

Fig. 7. Same as Fig. 3, but for $0.1<z \leq 0.15$.

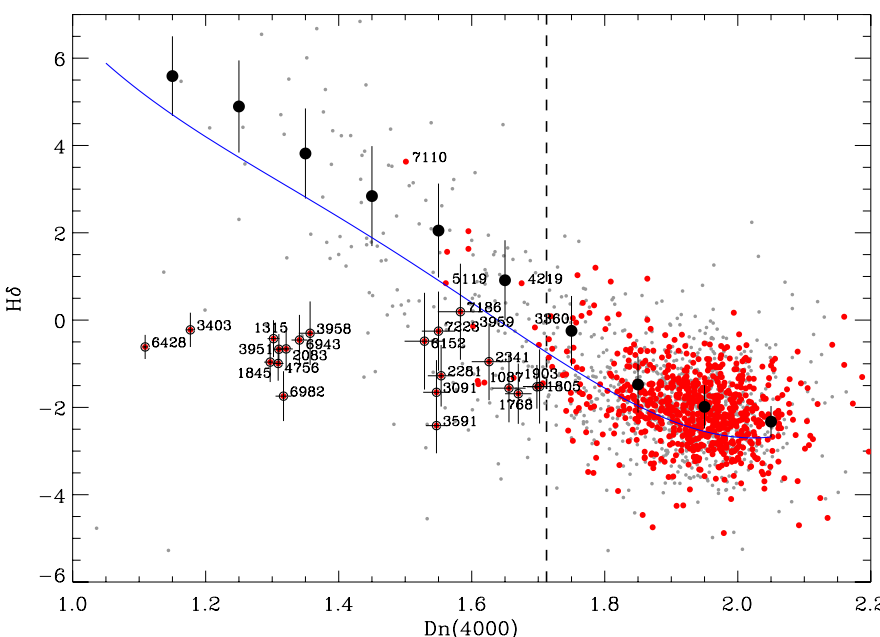

Fig. 8. Same as Fig. 4, but for $0.1<z \leq 0.15$.

candidates based on the Dn(4000) index alone. Their location in the Dn(4000) versus W2-W3 diagram suggests that these objects are low-luminosity LINERs and/or Seyfert galaxies (see Fig. 1). Including the MIR data thus substantially improves the purity of the resulting BL Lac sample.

\subsection{Properties of the selected objects}

Our method allowed us to isolate 36 BL Lac objects (or candidates). A detailed analysis of each individual source is deferred to a future paper. Nonetheless, we note that among the 15 new BL Lac candidates, ten are associated with an X-ray source according to the ASDC SED builder tool ${ }^{8}$, supporting their identification as genuine BL Lacs. Moreover, all but one object are massive elliptical galaxies, with stellar velocity dispersion $\sigma>160 \mathrm{~km} \mathrm{~s}^{-1}$, which means $\log \left(M_{\mathrm{BH}} / M_{\odot}\right)>7.8$ (Tremaine et al. 2002), i.e. the typical hosts of radio-loud AGNs. Their radio morphologies are consistent with those characterizing BL Lacs

\footnotetext{
8 http://tools.asdc.asi.it/SED/
}

(Antonucci \& Ulvestad 1985); i.e., they are dominated by a compact radio core in the FIRST images, and in some cases, they also show a one-sided jet or a halo.

We derive the radio-optical ${ }^{9}$ spectral index $\alpha_{\text {ro }}$ of the nonthermal emission for our candidates by estimating the jet optical contribution from the observed $\operatorname{Dn}(4000)$ value; i.e.

$F_{\mathrm{j}, \mathrm{o}}=\frac{\mathrm{D}_{\mathrm{n}}^{0}-\mathrm{Dn}(4000)}{\mathrm{D}_{\mathrm{n}}^{0}-1} F_{\mathrm{o}}$,

where $F_{\mathrm{o}}$ is the SDSS flux density (reddening-corrected) at $3900 \AA$. Figure 10 shows $\alpha_{\text {ro }}$ versus the logarithm of the radio luminosity of the FIRST central component $L_{\mathrm{r}}$. The mean value is $\left\langle\alpha_{\mathrm{ro}}\right\rangle=0.49$ and the standard deviation 0.08. The range of $\alpha_{\mathrm{ro}}$ covered by our objects overlaps that of the BL Lacs analyzed by Sambruna et al. (1996), Padovani et al. (2003), and Donato et al. (2001), going from $\sim 0.2$ to $\sim 0.75$.

Figure 11 shows the location of our BL Lac candidates in the WISE color-color diagram. They follow a sequence, starting from the region where passive elliptical galaxies are located (e.g., Wright et al. 2010; Raiteri et al. 2014), and moving toward the so-called WISE blazar strip identified by Massaro et al. (2011). We also show tracks corresponding to the addition of a power-law emission component $F_{v} \sim v^{-\alpha}$ of increasing strength to a $z=0.1$ elliptical galaxy flux. These tracks were obtained starting from the 13 Gyr elliptical galaxy template of the SWIRE Template Library ${ }^{10}$ (Polletta et al. 2007) for different values of $\alpha$ in the range $0-1$. They cover the region where most of our candidates are found. This supports the presence of a power-law emission component in the MIR bands, whose relative weight increases at longer wavelengths.

Overall, the properties of our candidates are reassuringly consistent with those of classical BL Lacs.

9 Estimated between $1.4 \mathrm{GHz}$ and $3900 \AA$, both in the source restframe; we adopted a null radio spectral index.

${ }^{10}$ http://www.iasf-milano.inaf.it/ polletta/templates/ swire_templates.html 


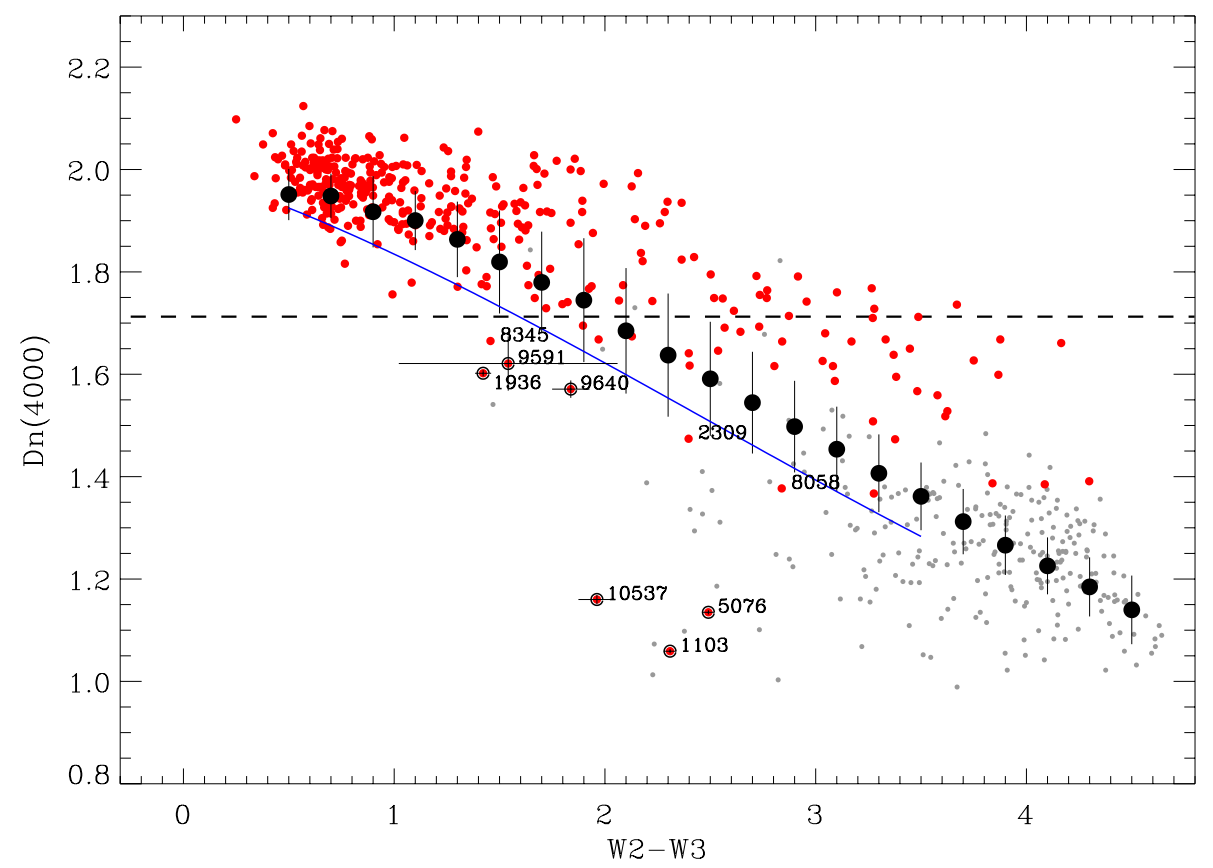

Fig. 9. Same as Fig. 3, but for the BH12-B sample at redshift $z \leq 0.15$.

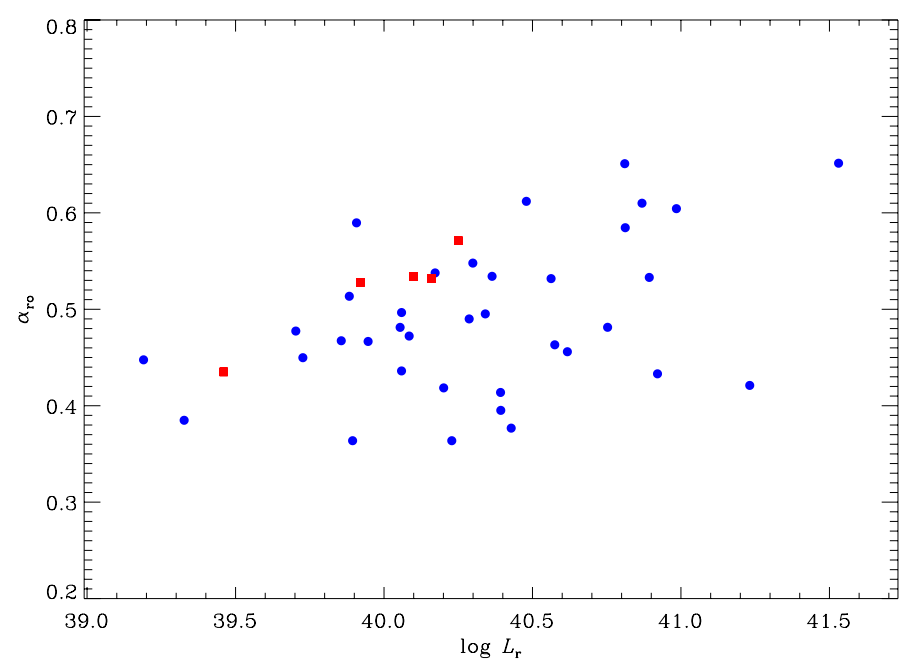

Fig. 10. Radio-optical spectral index $\alpha_{\text {ro }}$ versus the logarithm of the radio luminosity $L_{\mathrm{r}}$ for the $36 \mathrm{BL}$ Lac candidates in the forbidden zone of the $\mathrm{Dn}(4000)$ versus W2-W3 diagram (blue dots). The red squares correspond to the five objects located in the wedge region of the same diagram.

\section{Completeness of the low-luminosity BL Lacs sample}

Figure 12 shows the radio luminosity distribution of the FIRST central component, $L_{\mathrm{r}}$, for the $36 \mathrm{BL}$ Lacs identified with our method. They extend up to $\log L_{\mathrm{r}} \sim 41.5\left[\mathrm{erg} \mathrm{s}^{-1}\right.$ ]. The number of BL Lac candidates rapidly increases toward lower $L_{\mathrm{r}}$, reaching a peak at $\log L_{\mathrm{r}} \sim 40.5\left[\mathrm{erg} \mathrm{s}^{-1}\right]$ and then falls. This drop can be due to a genuine paucity of low-luminosity objects or, alternatively, to a decrease in the completeness of our sample. Before deriving the BL Lac radio luminosity function (RLF), we must consider the selection effects included in the various steps described in Sect. 3.

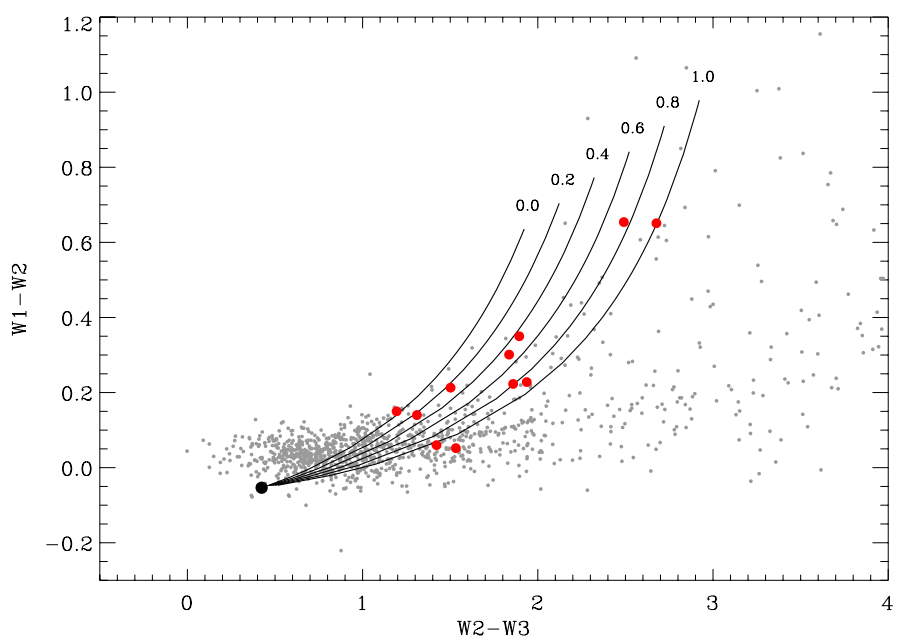

Fig. 11. WISE color-color diagram for the BH12 sample with $S / N(\mathrm{~W} 3)>2$ and $z \leq 0.1$ (gray dots). Red filled circles represent our BL Lac candidates in the same redshift range. The black tracks indicate the sequence followed by objects where a power-law component $F_{v} \sim v^{-\alpha}$ of increasing strength is added to the emission of an elliptical galaxy, represented by the black dot. This was derived from the SWIRE template of a 13 Gyr elliptical galaxy at $z=0.1$. Different tracks correspond to different values of $\alpha$ in the range $0-1$.

\subsection{Completeness of the NVSS-SDSS sample}

From the point of view of the radio completeness, BH12 included the sources with a NVSS flux density larger than $5 \mathrm{mJy}$. The vast majority of our selected BL Lac candidates have fluxes that are much larger than this limit, an indication that the flux threshold does not have a significant impact on our results. Nonetheless, we include the radio flux limit in the simulations presented in Sect. 5.

As for the optical selection of the sample, according to Montero-Dorta \& Prada (2009) the redshift completeness of the SDSS decreases with decreasing apparent magnitude, starting from $\sim 90 \%$ at the SDSS spectroscopic limit of $r=17.77$ and reaching $\sim 50 \%$ at $r=11.75$ (see Fig. 13). Most of the 


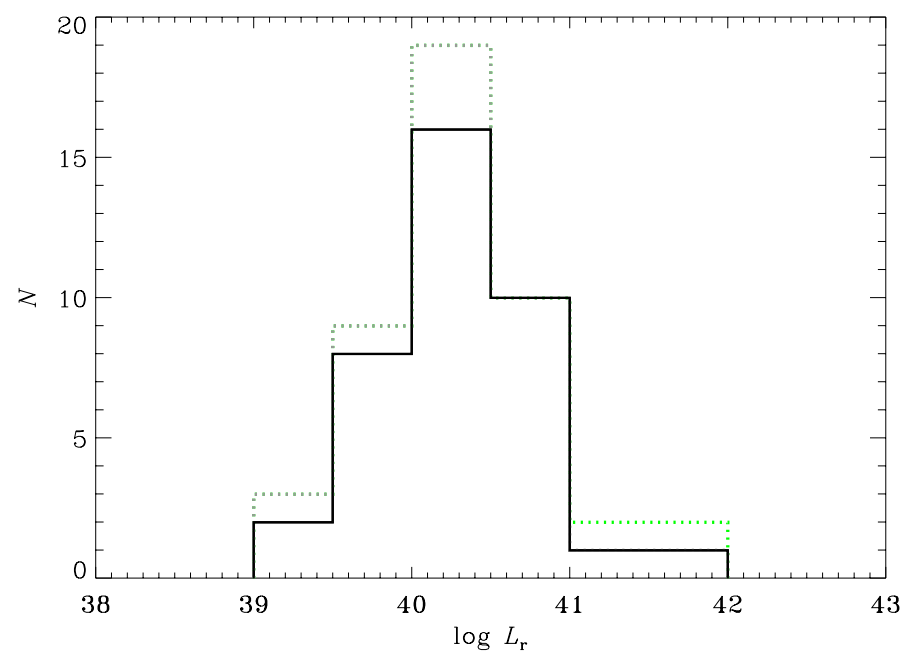

Fig. 12. Radio luminosity distribution at $1.4 \mathrm{GHz}$ of the $36 \mathrm{BL}$ Lac candidates. The dotted black histogram includes the five sources in the wedge, while the green one also considers the two BL Lacs tagged as STAR in the SDSS, see Sects. 4.3 and 4.4.

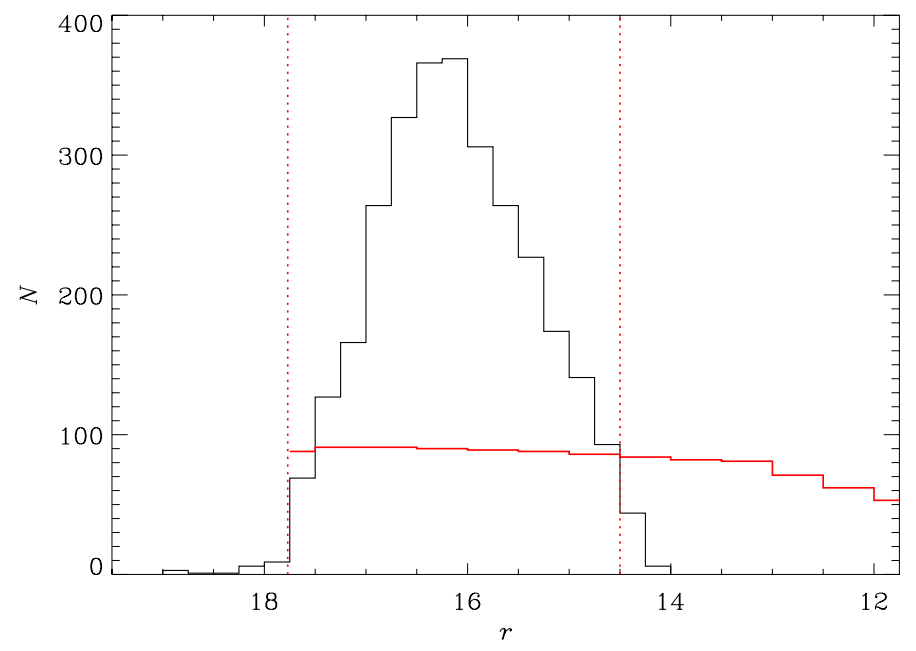

Fig. 13. Distribution of $r$ band magnitudes of the galaxies in the BH12-A sample with $0.05 \leq z<0.15$. The vertical dotted lines indicate the limits defining the SDSS main galaxies sample. The red histogram reports the SDSS completeness in percentage from Montero-Dorta \& Prada (2009).

incompleteness is due to the SDSS fiber cladding that prevents fibers on any given plate from being placed closer than $55^{\prime \prime}$ apart. This does not introduce a bias into the sample, but leads to a random loss of $\sim 10 \%$ (Zehavi et al. 2002) of the potential spectroscopic targets. For brighter (and more extended) objects, other effects become important, such as the superposition of bright saturated stars on the target.

Although the radio-loud AGN (RL-AGN) hosts are very luminous galaxies, it is important to assess their completeness level from the point of view of the SDSS spectroscopy. In Fig. 13 we show the distribution of $r$ band magnitudes of the galaxies in the BH12-A sample with $0.05 \leq z<0.15$. The distribution peaks at $r \sim 16.2$, and it rapidly falls on both sides. The number of objects is essentially reduced to zero before reaching the high and low magnitude limits imposed by their selection in the SDSS main galaxies sample ( $r=17.77$ and 14.5, respectively). This indicates that the selected sample of galaxies is complete.
Conversely, very nearby (and bright) galaxies might fall in the region of low completeness at low magnitudes. We then prefer to set a more stringent lower limit of $z=0.05$ on the galaxies considered. From the point of view of the BL Lac candidates, we discard only one of them (ID 1936), while from the point of view of the volume sampled, this is reduced by just $4 \%$.

\subsection{Check on the WISE constraints}

Could our requirements on the existence of a WISE counterpart and/or on the quality of WISE data affect the BL Lac candidates count? Among the 60 sources in the BH12 AGN sample without a WISE counterpart, there are no objects meeting our criteria to be considered BL Lac candidates: EW less than $5 \AA$, existence of a FIRST counterpart, and $\mathrm{Dn} \leq \mathrm{Dn}_{\max }$.

If we consider the 445 sources with $\mathrm{S} / N(\mathrm{~W} 3) \leq 2$ in the BH12-A sample, only three objects meet the selection criteria. However, the low quality of the overall multiband dataset for these sources suggests that we should not include them among the BL Lac candidates. In fact, not only are the WISE data very uncertain, but the optical indices also have large errors. We verified that among the 237 objects in the BH12-B sample without a WISE counterpart or with low S/N(W3), there are no BL Lac candidates.

\subsection{Are there BL Lacs outside the forbidden zone?}

The selection method we propose is based on the idea that the BL Lacs are found in the forbidden zone, while other radio emitting galaxies are located along a well-defined locus in the Dn(4000) vs. W2-W3 plane. However, there is the possibility that some BL Lacs follow this same sequence. Indeed, the simulated tracks presented in the right hand panel of Fig. 1 indicate that the objects with a spectral index in the MIR - optical bands $\gtrsim 1$ overlap with this region.

We then consider those sources that meet the same requirements as the ones used to select BL Lacs objects (rest-frame EW of the emission lines $<5 \AA$ and existence of a FIRST central counterpart) and that are located in the wedge region of the $\mathrm{Dn}(4000)$ vs. W2-W3 plane. This is the region below the $\mathrm{Dn}(4000)$ limit but above the polynomial fit to the $1 \sigma$ limit of the relationship derived for the MPA-JHU sample (see Fig. 3). We find 54 sources, 22 of which have $0.05 \leq z<0.15$. When discarding the $\mathrm{E}+\mathrm{A}$ galaxies, their number is reduced to ten. Five of them are spiral galaxies, not the typical BL Lac hosts, and they are most likely low-luminosity AGNs. The remaining five are elliptical galaxies: while one is a BZCAT object (probably a genuine BL Lac), the nature of the other four is highly uncertain. Their compact radio morphology does not provide us with any useful information. We list these objects in Table 2. They have Dn $(4000)$ values of 1.6-1.7 and W2-W3 colors in the range 1.7-2.1; i.e., they are all concentrated in a small portion of the wedge region, close to its apex. Their radio luminosity ranges from $\log L_{\mathrm{r}} \sim 39.5$ to $40.3\left[\mathrm{erg} \mathrm{s}^{-1}\right.$ ], see Fig. 12, and their $\alpha_{\text {ro }}$ goes from 0.44 to 0.57 (see Fig. 10).

\subsection{An external completeness test: the BZCAT}

We here assess the completeness of our sample against the compilation of BL Lac objects (or candidates) included in the multifrequency catalog of blazars Roma-BZCAT. The edition 4.1.1 (August 2012) contains 3149 blazars, 1221 of which are BL Lac objects or candidates (BZB), and 221 are blazars of uncertain 

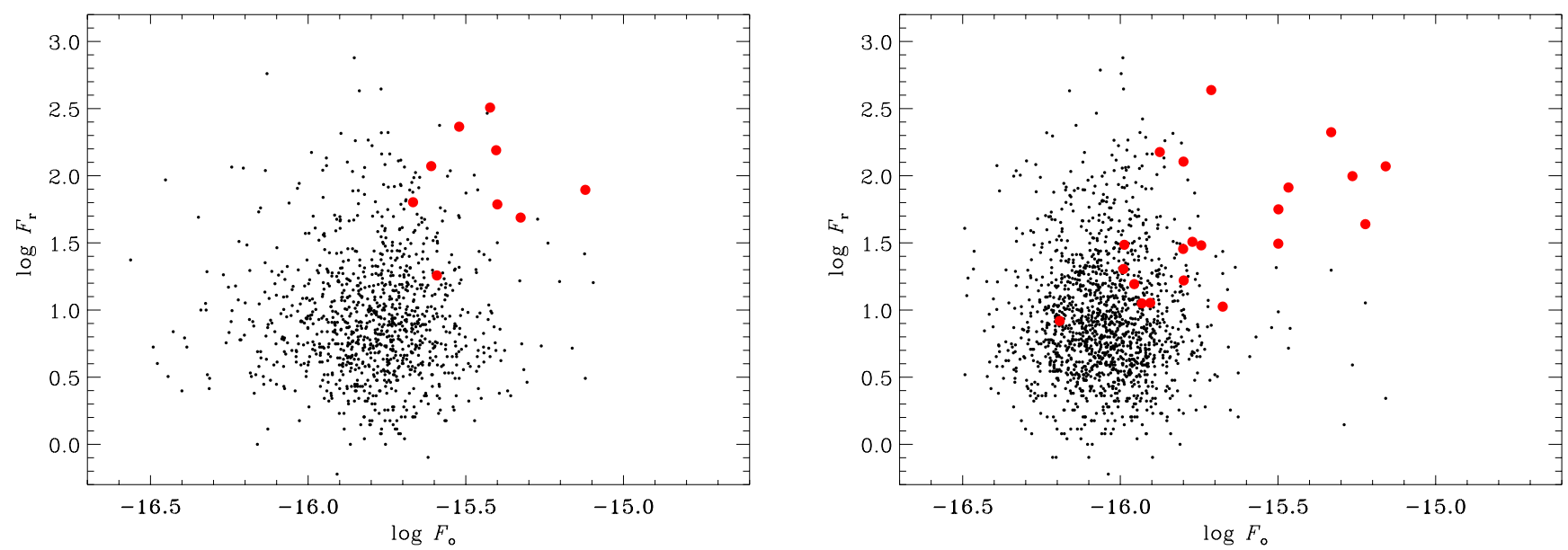

Fig. 14. Left panel: the flux density of the FIRST central component, $F_{\mathrm{r}}$, versus dereddened flux density within the SDSS fiber at $3900 \AA$ A $F_{\mathrm{o}}$, for the sources with $0.05<z \leq 0.1$. The candidate BL Lacs are plotted with red circles. Right panel: same as in the left panel, but for $0.1<z \leq 0.15$.

Table 2. Possible BL Lacs in the BH12 sample with $0.05<z \leq 0.15$ in the wedge region of the Dn(4000) versus W2-W3 diagram.

\begin{tabular}{rrrcccccc}
\hline \hline ID & RA & Dec & W2-W3 & Dn(4000) & $z$ & $F_{1.4 \mathrm{GHz}}$ & $\log L_{\text {radio }}$ & BZCAT \\
\hline 935 & 147.13342 & 55.59314 & 2.064 & 1.610 & 0.118 & 28.6 & 40.16 & BZUJ0948+5535 \\
3062 & 194.97218 & 57.86380 & 1.711 & 1.707 & 0.149 & 9.9 & 39.92 & - \\
5525 & 213.01450 & 29.46714 & 1.970 & 1.619 & 0.115 & 37.0 & 40.25 & - \\
2468 & 226.09259 & 47.68667 & 1.957 & 1.721 & 0.093 & 9.5 & 39.46 & - \\
1278 & 359.67532 & -9.90999 & 2.146 & 1.663 & 0.105 & 31.8 & 40.10 & - \\
\hline
\end{tabular}

Notes. Column description: 1) ID number; 2) RA [deg]; 3) Dec [deg]; 4) W2-W3; 5) Dn(4000); 6) redshift; 7) flux density at 1.4 GHz of the FIRST central component [mJy]; 8) the corresponding luminosity [ $\left.\mathrm{erg} \mathrm{s}^{-1}\right]$; 9) BZCAT name.

type (BZU). Such objects have been selected with different methods and from observations in various bands. The comparison might unveil the presence of bias in our selection method. Details on the cross-match between the BZCAT sources and our sample are given in Appendix A.

In summary, in the $0.05<z \leq 0.1$ redshift range, the comparison between the BH12 sample and BZCAT suggests that we did not miss any object because of our selection method. We only lost 3 objects out of 18 because of fiber collision, which does not, however, introduce any bias into the sample but only a lower effective sky coverage.

Moving to higher redshift, we recover all BZB and lineless BZU with the exception of six objects: two are bright featureless sources that are not included in the BH12 sample because they are tagged as STAR. We consider them in the RLF discussion. One object was instead missed because it lies in the wedge region of our Dn(4000) versus W2-W3 diagnostic plane. As shown in Fig. 1, this is the region populated by sources with MIR-optical spectral index $\alpha \sim 1$, so likely LBL objects. Finally, three were discarded by our method because of a high Dn(4000) value, between 1.95 and 2.01. However, two of them also have WISE colors that are typical of quiescent galaxies, W2-W3 = 0.76 and 0.39 , respectively. For one of them, as detailed in the Appendix, we have strong evidence that it might be an incorrect identification.

\section{BL Lac selection function}

As discussed in Sect. 3, the main requirement for including an object belonging to the NVSS-SDSS sample in our candidates list is a flux contribution from the non-thermal jet component, $F_{\mathrm{j}, \mathrm{o}}$, of at least one-third of the galactic one at $3900 \AA$ (rest frame).
To estimate how this criterion is reflected in the selection function at different values of radio luminosity, we ran a Monte Carlo simulation. In each $L_{\mathrm{r}}$ bin, we extracted 100000 objects with:

i) a random distribution in redshift between $0.05<z \leq 0.15$, properly weighting for the appropriate volumes and assuming a constant source density with redshift, to derive the corresponding radio flux; objects with radio flux density below the $5 \mathrm{mJy}$ threshold of the BH12 sample are discarded;

ii) a random spectral index $\alpha_{\text {ro }}$ (see below for a detailed discussion on this parameter) that leads to an estimate, for each object, of the expected jet optical emission $F_{\mathrm{j}, \mathrm{o}}^{\prime}$ $\left[\operatorname{erg~s}^{-1} \mathrm{~cm}^{-2} \AA^{-1}\right]$ as

$\log F_{\mathrm{j}, \mathrm{o}}^{\prime}=\log F_{\mathrm{r}}[\mathrm{mJy}]-5.74 \alpha_{\mathrm{ro}}-14.71$;

iii) a random value of the flux density at $3900 \AA$ (rest frame) with average and dispersion measured from the sample galaxies divided in redshift bins of 0.01. In Fig. 14 we show the distribution of radio versus optical fluxes into two redshift ranges.

For each object we tested whether the ratio between jet and galactic optical emission is above the $f=1 / 3$ threshold. The fraction of objects expected to be selected as BL Lac candidates as a function of radio luminosity can finally be derived.

The assumption on the distribution of the radio-optical spectral index is the key to this simulation. In Sect. 3.3 we estimated a mean value of $\left\langle\alpha_{\text {ro }}\right\rangle=0.49 \pm 0.08$. However, our results might be plagued by selection effects, since high $\alpha_{\text {ro }}$ objects of low radio luminosity could escape detection in the optical band. To assess how robust our estimates are, we compared 


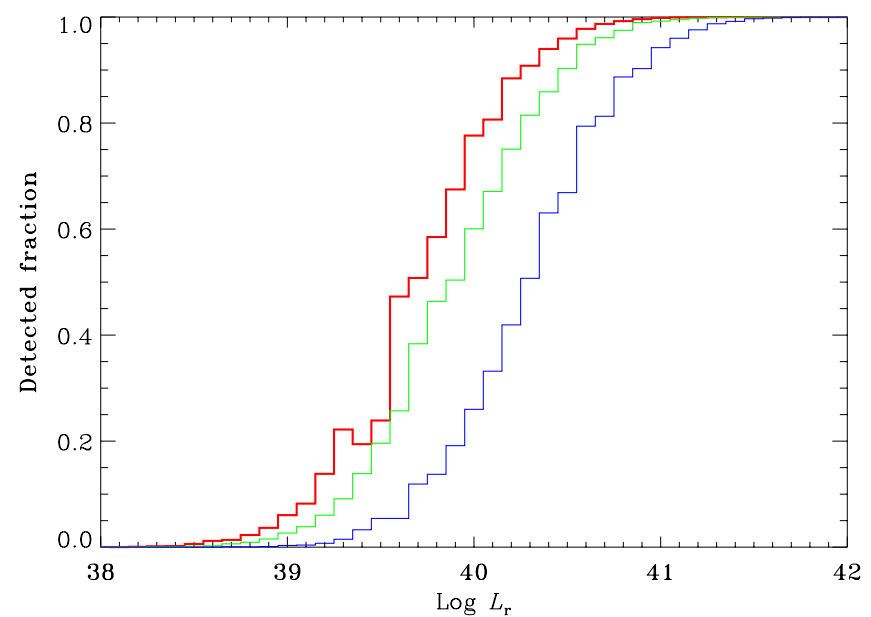

Fig. 15. Expected fraction of BL Lac candidates recovered with our selection method at various levels of radio luminosity obtained with Monte Carlo simulations. The various lines refer to different assumptions on the radio-optical spectral index. Red: $\alpha_{\mathrm{ro}}=0.49$, the mean obtained from the $35 \mathrm{BL}$ Lac candidates with $0.05<z \leq 0.15$; green: $\alpha_{\mathrm{ro}}=0.53$, the mean of the candidates with $\log L_{\mathrm{r}}>40.5 \mathrm{erg} \mathrm{s}^{-1}$; blue: $\alpha_{\mathrm{ro}}=0.59$, the mean of the 1Jy BL Lacs.

them to those available in the literature. The average spectral index of the (radio-selected) 1 Jy sample of BL Lacs (Stickel et al. 1991) is $\left\langle\alpha_{\text {ro }}\right\rangle=0.59$ with a dispersion of 0.10 (Sambruna et al. 1996). Adopting their average $\alpha_{\text {ro }}$ for the simulation, we obtain the selection function reproduced as the blue curve in Fig. 15: it reaches $80 \%$ at $\log L_{\mathrm{r}} \sim 40.5\left[\mathrm{erg} \mathrm{s}^{-1}\right.$ ]. This means that, at least above this threshold, the BL Lacs sample we selected is very close to completeness and that the distribution of $\alpha_{\text {ro }}$ is not significantly affected by selections biases. Its average, $\left\langle\alpha_{\text {ro }}\right\rangle=0.53 \pm 0.08$, can be considered as a robust value. The selection function obtained by adopting this value is shown in Fig. 15.

The 1 Jy objects are, in general, much brighter than the BL Lacs we are considering, with radio luminosities reaching $10^{45} \mathrm{erg} \mathrm{s}^{-1}$. If we limit to the $1 \mathrm{Jy}$ sources with $L_{\mathrm{r}}<10^{43} \mathrm{erg} \mathrm{s}^{-1}$ we obtain $\left\langle\alpha_{\text {ro }}\right\rangle=0.52 \pm 0.09$, which agrees remarkably with the value quoted above, while the average $\alpha_{\text {ro }}$ for those with $L_{\mathrm{r}}>10^{43} \mathrm{erg} \mathrm{s}^{-1}$ is $0.65 \pm 0.08$. These results suggest there is a trend between $\alpha_{\text {ro }}$ and the radio luminosity, as expected from the so-called blazar sequence (Fossati et al. 1998). This would account for the (slightly) lower average value we find for our LPBL sample. Indeed, the sources of the $1 \mathrm{Jy}$ sample (limiting to the 22 with measured redshift) show a linear correlation between $\alpha_{\text {ro }}$ and $L_{\mathrm{r}}$, with a slope of 0.06 . The Spearman rank correlation test returns a probability of only $2 \%$ that the two variables are not correlated. We therefore repeat the simulation by adopting the observed distribution of spectral indices; i.e., $\left\langle\alpha_{\text {ro }}\right\rangle=0.49$ (Fig. 15). The selection function obtained with this assumption differs only marginally from what is derived for $\left\langle\alpha_{\mathrm{ro}}\right\rangle=0.53$, with a horizontal shift of only $\sim 0.2$ dex.

\section{The BL Lacs luminosity function}

Several studies have dealt with the determination of the BL Lacs RLF. In all cases, the approach was based on flux-limited samples. In our study, instead, we consider an essentially volumelimited sample, because the radio, optical, and MIR flux limits do not represent a significant constraint in our selection. The advantage of this method is twofold: all objects have a known

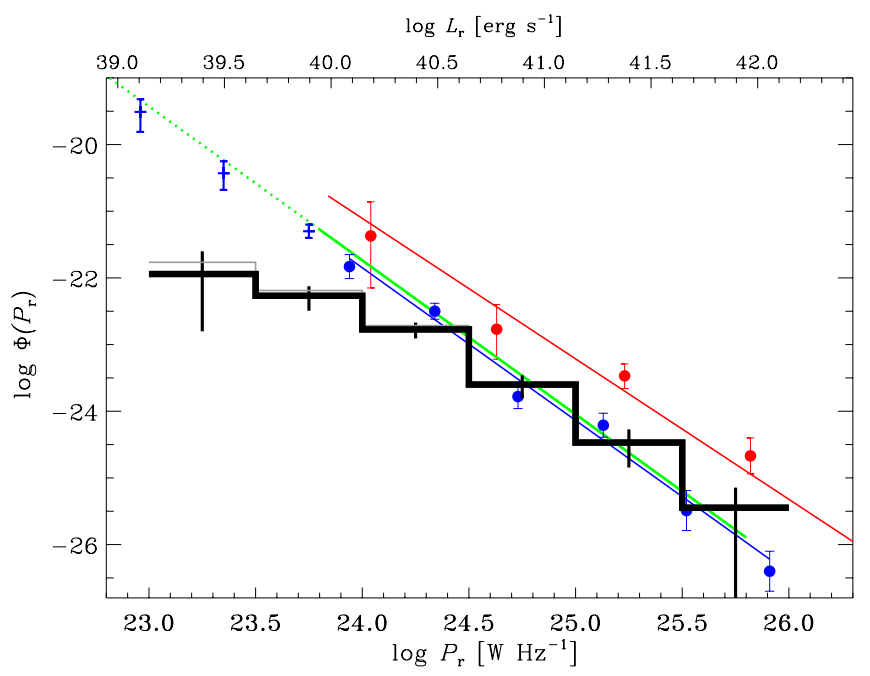

Fig. 16. RLF at $5 \mathrm{GHz}$ of the selected BL Lac candidates (black histogram). The gray histogram shows the effects of including the five objects located in the wedge. The results from Padovani et al. (2007) are in red, while blue dots refer to the work by Marchã \& Caccianiga (2013) for classical BL Lacs. We also report (as plus signs) three points at low luminosity of the Marchã \& Caccianiga RLF for Type 0 AGNs. The green line is the RLF normalized to match the number of objects of our sample with $\log P_{\mathrm{r}}>23.8\left[\mathrm{~W} \mathrm{~Hz}^{-1}\right]$, after having adopted the same slope of Marchã \& Caccianiga (2013).

redshift, and they are all within a very limited range, so that evolution can be neglected.

We can then derive the BL Lacs RLF by simply dividing the number of detected objects by the total volume covered, recalling that the intersection between the spectroscopic DR7 and FIRST covers $17.3 \%$ of the whole sky. By also considering the effects of fiber cladding, this results in a volume of $0.158 \mathrm{Gpc}^{3}$ from $z=0.05$ to $z=0.15$. We convert the radio luminosity distribution shown in Fig. 12 (including the 35 candidates with $0.05<z \leq 0.15$ and the two objects tagged as STAR) by dividing the number of objects by the radio luminosity, the selection function, and the total volume. The errors on the RLF have been estimated by combining Poisson statistics with the uncertainties related to the selection function ${ }^{11}$. The result is shown in Fig. 16. To ease the comparison with previous studies, we present the radio luminosities in both cgs and MKS units and assume a zero radio spectral index to convert from 1.4 to $5 \mathrm{GHz}$ (Stickel et al. 1991).

We can compare our results on the RLF with those obtained by previous works and, in particular, with Padovani et al. (2007) and Marchã \& Caccianiga (2013). We assume a RLF in the form of a power law, $\Phi\left(P_{\mathrm{r}}\right) \propto P_{\mathrm{r}}^{-B}$. Since our sample covers a limited range of radio luminosities, we refrain from calculating the RLF slope. If we adopt a power-law slope of $B=2.31$ as in Marchã \& Caccianiga (2013) for classical BL Lacs, we can calculate the normalization of our RLF so that the integrated RLF returns the number of observed objects above $\log P_{\mathrm{r}}=23.8\left[\mathrm{~W} \mathrm{~Hz}^{-1}\right]$, the range of powers common to both studies. This is displayed in Fig. $16^{12}$. The comparison shows very good agreement with the results obtained by Marchã \& Caccianiga for BL Lacs and

11 We considered the two assumptions: $\left\langle\alpha_{\text {ro }}\right\rangle=0.49$ and 0.53 . The differences in the resulting RLF are of only $0.17,0.15$, and 0.05 dex for the three bins at the lowest luminosities. By adopting instead $\left\langle\alpha_{\mathrm{ro}}\right\rangle=0.59$, these bins are further increased by $0.75,0.38$, and 0.12 dex, respectively. 12 Had we used $B=2.12$ as derived by Padovani et al. (2007), we would have obtained a difference of only 0.08 dex at $\log P_{\mathrm{r}}=24.5\left[\mathrm{~W} \mathrm{~Hz}^{-1}\right]$. 


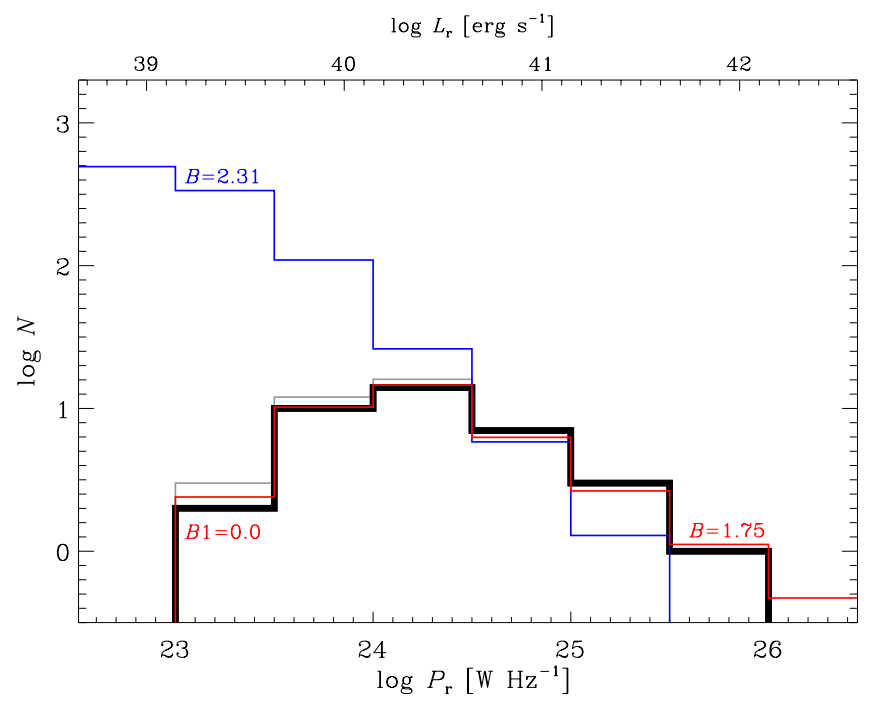

Fig. 17. Observed number of BL Lacs (black solid line) versus radio power. The blue histogram represents the predicted number of objects in our volume extrapolating the RLF derived for our sample to low radio luminosities (green line in Fig. 16) and multiplied by the selection function (red line in Fig. 15). The gray histogram shows the effects of the inclusion of the five objects located in the wedge. The red histogram displays the same quantity as for a RLF with $B=1.75$ and with a break at $\log P_{\mathrm{r}}=24.5\left[\mathrm{~W} \mathrm{~Hz}^{-1}\right]$, below which the RLF slope is $B 1=0$.

a mismatch between our RLF and the Padovani et al. one by a factor $\sim 3$.

The main aim of our study is to isolate and analyze the least luminous BL Lacs. Indeed, we detected candidates down to $\log P_{\mathrm{r}} \sim 23\left[\mathrm{~W} \mathrm{~Hz}^{-1}\right]$. We defer a detailed study of the individual objects and of their SED to a future paper. Here we want to address the behavior of their RLF in a poorly studied regime of low radio power. The only study reaching these luminosities is from Marchã \& Caccianiga (2013): they consider not only classical BL Lacs but also the objects they defined as Type 0 and weaklined AGNs. Their space density is substantially higher than for our BL Lac sample, with, say, about ten times more Type 0 AGNs than BL Lacs between $\log P_{\mathrm{r}}=23.5$ and $24.0\left[\mathrm{~W} \mathrm{~Hz}^{-1}\right]$. However, recent studies have shown that the dominant population of radio galaxies in the local Universe is formed by the socalled FR 0 sources (Baldi \& Capetti 2010; Sadler et al. 2014; Baldi et al. 2015). Their lines EW are generally low (more than $80 \%$ showing values lower than $5 \AA$, R. Baldi, private communication). Moreover, they are generally sources with a high core dominance and, for this reason, often flat spectrum radio sources. As explained by Baldi et al. (2015) the deficit of extended radio emission is an intrinsic property of FR 0 , and it is not due to a beaming effect of orientation, as in the case of BL Lacs. In other words, a significant fraction of the FR 0 population conforms to the definition of Type 0 and weak-lined AGNs. Therefore, the association of all Type 0 and weak-lined AGNs with LPBL might not be justified.

We estimate the predicted number of LPBL in our volume by extrapolating the RLF derived above to low powers, taking our selection function into account (see Fig. 15). The result is shown in Fig. 17, where we compare the observed and predicted number counts. A large disagreement emerges, where the difference is a factor of $\sim 10$ already at $\log P_{\mathrm{r}} \sim 10^{23.75} \mathrm{~W} \mathrm{~Hz}^{-1}$ (i.e., $\log L_{\mathrm{r}} \sim 40$ ), where our selection function is high (around 70\%) enough to make this result robust.

We then consider the possibility of a break in the RLF occurring at $P_{\text {break }}$. Furthermore, it appears that also at high luminosities the slope might be less than the value of 2.31 adopted. We are not in the position of accurately determining all parameters describing the RLF with the available data. Nonetheless, in Fig. 17 we show an illustrative example of a RLF with $B=1.75$, a break at $\log P_{\text {break }}=24.5\left[\mathrm{~W} \mathrm{~Hz}^{-1}\right]$, and a slope below the break $B 1=0$, which fairly matches the observed counts at all luminosities. This result is effectively independent of the assumption used for the radio-optical spectral index: had we used the average value derived for the $1 \mathrm{Jy}$ sample, the break luminosity would have decreased by just a factor $\sim 1.7$.

Values of $B 1$ below 1, as suggested by this analysis, correspond to BL Lacs number counts growing with luminosity up to $P_{\text {break }}$, implying the existence of a peak.

\subsection{The BL Lac RLF and the AGN unified model}

According to the AGN unified scheme, the RLFs of BL Lacs and RL-AGNs are expected to be linked to each other. In fact, blazars are thought to be the beamed version of radio galaxies (see, e.g., Urry \& Padovani 1995). The effect of relativistic beaming on the RLF was analyzed in a series of papers by Urry \& Shafer (1984), Urry \& Padovani (1991), Urry et al. (1991), and Urry \& Padovani (1995). If the RLF of the parent population of unbeamed objects is described by a broken power law, the break will produce a change of slope in the BL Lac RLF at a power amplified by $\sim \delta_{\max }^{3}$, where $\delta_{\max }$ is the maximum value of the jet Doppler factor distribution ${ }^{13}$.

The RLF of RL-AGNs extending to the lowest value of radio luminosity has been obtained by Mauch \& Sadler (2007), covering the range $\log P_{\mathrm{r}}=20.4-26.4\left[\mathrm{~W} \mathrm{~Hz}^{-1}\right]$. At low luminosities, the RLF does not significantly depart from a power law, and their results suggest that any break must be located below $\log P_{\mathrm{r}} \sim 21.6 \mathrm{~W} \mathrm{~Hz}^{-1}$, where the limited number of observed objects makes the RLF shape uncertain. On the other hand, the number density of radio emitting AGNs cannot exceed that of their potential hosts: this argument has been used by Capetti \& Raiteri (2015), requiring a minimum black hole mass of $\log \left(M_{\mathrm{BH}} / M_{\odot}\right)>7.5$ for the host of RL-AGNs. With this approach they found that the break in the RLF of RL-AGNs must be located at $P_{\mathrm{r}} \gtrsim 10^{20.5} \mathrm{~W} \mathrm{~Hz}^{-1}$.

The ratio between the break powers in the RLFs of BL Lacs and RL-AGNs is a factor $\sim 10^{3}-10^{4}$. If adopting $p=3$, this suggests a value of the Doppler factor $\delta_{\max } \sim 10-20$, consistent with the typical results from the observations and the model predictions (e.g., Ghisellini et al. 1993; Savolainen et al. 2010).

\section{Summary and conclusions}

In this paper we presented a new strategy for recognizing BL Lac objects, based on the combination of optical indices derived from the SDSS spectra with MIR colors from the AllWISE survey. This enabled us to separate BL Lac objects from lowluminosity AGNs and star-forming galaxies. Our method is particularly useful for identifying LPBL, where the traditional optical non-thermal signature is reduced thanks to the dominant host-galaxy emission. We applied it to the sample of 18286 radio sources selected by BH12 by combing the SDSS DR 7 spectra with the NVSS and FIRST surveys. We showed that in the Dn(4000) versus W2-W3 plane, BL Lacs fall in a region (defined as forbidden zone) that is scarcely populated

${ }^{13}$ The Doppler factor $\delta$ is defined as $\delta=[\Gamma(1-\beta \cos \theta)]^{-1}$, where $\Gamma=\left(1-\beta^{2}\right)^{-1 / 2}$ is the bulk Lorentz factor, $\beta$ is the velocity in units of the speed of light, and $\theta$ the viewing angle. The exact value of the $\delta$ exponent depends on the geometry of the emitting region. 
by the general galaxies population. The main contaminants are the A+E (post-starburst) galaxies that can be easily removed with the aid of their characteristic pronounced Balmer absorption lines. When considering low luminosities, the use of the $\operatorname{Dn}(4000)$ index alone is not sufficient to obtain a clean sample. In fact, the constraint based on the W2-W3 color allowed us to remove 54 objects (most of which are low-luminosity LINERs and/or Seyfert galaxies) that would instead be selected as BL Lac candidates. Including the MIR data thus substantially improves the quality of the resulting BL Lac sample.

We isolated $36 \mathrm{BL}$ Lac candidates up to $z=0.15$. All but one are associated with luminous elliptical galaxies with radio powers ranging from $\log L_{\mathrm{r}}=39.2$ to 41.5 [ $\mathrm{erg} \mathrm{s}^{-1}$ ]. Fifteen of the selected objects are new identifications, most of which have an X-ray counterpart.

We discussed various issues concerning our sample completeness. The radio flux threshold does not have a significant impact on our results. As for the optical selection, most of the expected RL-AGN hosts are included in the MPA-JHU catalog. The only exceptions are the low-luminosity tail close to the upper redshift boundary and the most luminous galaxies located at very low redshift. We then set a more stringent lower limit of $z=0.05$ on the galaxies considered. We used the BZCAT for an external check of our results. We recovered all the known objects in the catalog up to $z=0.1$, while we lost less than $10 \%$ of them in the $0.1<z<0.15$ range. In particular, only two very bright BL Lacs are lost because of their point-like appearance, which caused these objects to be misclassified as STAR in the SDSS. We also tested the effects of the criterion based on the W2-W3 threshold. Out of the 54 sources mentioned above, we find five objects that conform to the general properties of BL Lacs (since hosted in elliptical galaxies and having low lines EW). However, they are of uncertain nature because they lie in a region of the W2-W3 vs. Dn(4000) plane populated by other classes of AGNs.

We simulated the selection function of BL Lacs within our sample. The key ingredient is the assumption on the distribution of the radio-to-optical spectral index for which we adopted the values derived from our candidates. Since they agree with those found in the literature, our results are not significantly affected by selections biases. The sample completeness reaches $\sim 50 \%$ at $\log L_{\mathrm{r}} \sim 39.7\left[\mathrm{erg} \mathrm{s}^{-1}\right]$.

The selected BL Lac candidates were used to explore the BL Lacs RLF at low radio powers. Since we are dealing with an essentially volume-limited sample at low redshift, we can neglect evolutionary effects on the RLF. This can be obtained simply by counting all objects at the various radio luminosities within the considered volume. We found good agreement with the results by previous authors for $\log P_{\mathrm{r}}>24.5\left[\mathrm{~W} \mathrm{~Hz}^{-1}\right]$.

However, we found a dramatic paucity of LPBL with respect to the extrapolation of the BL Lacs RLF, a result that is altered neither by the inclusion of the five potential BL Lacs nor by the uncertainties on the selection function. This occurs well above the power at which our selection function falls significantly and requires a break in the RLF. We estimated that the break is located at $\log P_{\mathrm{r}} \sim 24.5\left[\mathrm{~W} \mathrm{~Hz}^{-1}\right]$. The slope of the RLF below the break is consistent with a zero value, implying the presence of a peak in the number density distribution of BL Lacs.

In the framework of the AGN unification scheme, BL Lacs are the beamed counterparts of low-power RL-AGNs, so that a link between their RLFs is expected. In particular, a low-power break in the parent population translates into a change in slope in the RLF of BL Lacs. Indeed, there is evidence of a lowluminosity break in the RL-AGN RLF located about three to four orders of magnitude below what we found for BL Lacs. This corresponds to a maximum Doppler factor of $\sim 10-20$, in agreement with the observations and model predictions.

In the near future, we plan to do a detailed analysis of the whole BL Lac candidate sample derived in this paper with two aims: i) to confirm their nature as genuine BL Lacs and ii) to characterize their broad-band properties. In particular, we need to obtain high-resolution radio data in order to isolate their nuclear emission and X-ray data to explore the high-energy nonthermal emission.

Acknowledgements. We are deeply indebted to the anonymous referee for the careful reading of the manuscript and for the many useful and insightful suggestions. This research made use of the NASA/ IPAC Infrared Science Archive and Extragalactic Database (NED), which are operated by the Jet Propulsion Laboratory, California Institute of Technology, under contract with the National Aeronautics and Space Administration. Funding for SDSS-III has been provided by the Alfred P. Sloan Foundation, the Participating Institutions, the National Science Foundation, and the US Department of Energy Office of Science. The SDSS-III web site is http://www.sdss3.org/. SDSS-III is managed by the Astrophysical Research Consortium for the Participating Institutions of the SDSS-III Collaboration, including the University of Arizona, the Brazilian Participation Group, Brookhaven National Laboratory, University of Cambridge, Carnegie Mellon University, University of Florida, the French Participation Group, the German Participation Group, Harvard University, the Instituto de Astrofisica de Canarias, the Michigan State/Notre Dame/JINA Participation Group, Johns Hopkins University, Lawrence Berkeley National Laboratory, Max Planck Institute for Astrophysics, Max Planck Institute for Extraterrestrial Physics, New Mexico State University, New York University, Ohio State University, Pennsylvania State University, University of Portsmouth, Princeton University, the Spanish Participation Group, University of Tokyo, University of Utah, Vanderbilt University, University of Virginia, University of Washington, and Yale University. This publication makes use of data products from the Two Micron All Sky Survey, which is a joint project of the University of Massachusetts and the Infrared Processing and Analysis Center/California Institute of Technology, funded by the National Aeronautics and Space Administration and the National Science Foundation. Part of this work is based on archival data, software or online services provided by the ASI Science Data Center (ASDC).

\section{Appendix A: Details of the comparison with BZCAT}

In the BZCAT list there are $34 \mathrm{BZB}$ with $0.05<z \leq 0.1$, nine of which are located in the area covered by both FIRST and the spectroscopic DR 7. Seven of them are in our list of BL Lacs candidates. The remaining two are:

- BZBJ1216+0929, which was selected as spectroscopic target, but its spectrum was not obtained owing to a fiber collision; and

- BZBJ1426+2415, which shows a featureless spectrum; the lack of any signature from the galaxy emission cast doubts on the accuracy of its tentative redshift estimate, $z=0.055$; indeed, the DR 7 value is $z=2.243$.

A check on the BZU is more problematic, because most of them are FSRQs. There are 24 BZU in BZCAT at $0.05<z \leq 0.1$. Nine of them are in our survey area, but two lack spectra because of fiber collision. The other seven have strong emission lines.

In the redshift range $0.1<z \leq 0.15$, we found $59 \mathrm{BZB}$ in BZCAT, 26 of which are located in the area covered by both the spectroscopic DR7 and FIRST. When excluding three objects that have no spectrum because of fiber collision and one object whose spectrum has a defect in the $\mathrm{H} \alpha$ region, we are left with 22 sources, 15 of which belong to the BH12 sample. The other seven are lost because:

- one object has no spectrum because it is too faint, but we know from BOSS that it is a spiral galaxy; 
- three are featureless objects whose SDSS redshift is higher than 0.15 ;

- one is a strong-lined object reaching an EW of $\sim 10 \AA$;

- two (BZBJ0809+5218 and BZBJ1221+2813-W Comae) are classified as STARS in the SDSS and thus do not enter in the main galaxy sample analyzed by $\mathrm{BH} 12$; they have featureless spectra, and $\log L_{\mathrm{r}}=41.11$ and 41.54 , respectively.

Among the 15 sources included in the BH12 sample, 13 are in our list of BL Lacs candidates, while two sources (BZBJ0810+4911 and BZBJ1404+2701) were not selected by our method because of a high $\operatorname{Dn}(4000)$. They have $\log L_{\mathrm{r}}=$ 39.50 and 40.39 , respectively. The first one was identified as a BL Lac because of $\gamma$-ray emission detected by the EGRET instrument on-board the Compton Gamma-Ray Observatory, but it is not present in the third Fermi Large Area Telescope source catalog. Furthermore, Liuzzo et al. (2013) did not detect it with the VLA at $8.4 \mathrm{GHz}$, so we consider it a dubious association.

The above analysis leads to the conclusion that in the $0.1<$ $z \leq 0.15$ redshift range, our selection method recovered all BZB but two sources characterized by a high Dn(4000) value. Another two objects were lost because they are so bright that they are not classified as galaxies and so do not enter the BH12 sample. Another four objects lack complete spectral information.

Among the BZU, there are 23 objects in BZCAT at $0.1<$ $z \leq 0.15,14$ that are in the region covered by both the spectroscopic DR7 and FIRST. One object has no spectrum because of fiber collision, two have strong emission lines, 11 are in the BH12 sample, but only three have rest-frame EWs less than $5 \AA$ and only one, BZUJ0936+0509, is selected by our method (Table 1). The other two are BZUJ0820+4853 and BZUJ0948+5535. The former was discarded because of a high $\operatorname{Dn}(4000)$ of 2.01, while the latter lies in the wedge region of Fig. 7. They have $\log L_{\mathrm{r}}=40.65$ and 40.12 , respectively.

The analysis of the BZU in the $0.1<z \leq 0.15$ redshift range thus reveals that our methods did not recover two sources out of 14. One other object was missed because of fiber collision.

\section{References}

Abdo, A. A., Ackermann, M., Ajello, M., et al. 2010, ApJ, 708, 1310 Aller, M. F., Aller, H. D., \& Hughes, P. A. 1996, in Blazar Continuum Variability, eds. H. R. Miller, J. R. Webb, \& J. C. Noble, ASP Conf. Ser., 110, 193 Antonucci, R. R. J., \& Ulvestad, J. S. 1985, ApJ, 294, 158
Baldi, R. D., \& Capetti, A. 2010, A\&A, 519, A48

Baldi, R. D., Capetti, A., \& Giovannini, G. 2015, A\&A, 576, A38

Balogh, M. L., Morris, S. L., Yee, H. K. C., Carlberg, R. G., \& Ellingson, E. 1999, ApJ, 527, 54

Best, P. N., \& Heckman, T. M. 2012, MNRAS, 421, 1569 (BH12)

Brinchmann, J., Charlot, S., White, S. D. M., et al. 2004, MNRAS, 351, 1151

Capetti, A., \& Raiteri, C. M. 2015, MNRAS, 449, 128

Donato, D., Ghisellini, G., Tagliaferri, G., \& Fossati, G. 2001, A\&A, 375, 739

Fossati, G., Maraschi, L., Celotti, A., Comastri, A., \& Ghisellini, G. 1998, MNRAS, 299, 433

Ghisellini, G., Padovani, P., Celotti, A., \& Maraschi, L. 1993, ApJ, 407, 65

Giommi, P., Padovani, P., Polenta, G., et al. 2012a, MNRAS, 420, 2899

Giommi, P., Polenta, G., Lähteenmäki, A., et al. 2012b, A\&A, 541, A160

Goto, T., Nichol, R. C., Okamura, S., et al. 2003, PASJ, 55, 771

Kellermann, K. I., Lister, M. L., Homan, D. C., et al. 2004, ApJ, 609, 539

Konigl, A. 1981, ApJ, 243, 700

Landt, H., Padovani, P., \& Giommi, P. 2002, MNRAS, 336, 945

Liuzzo, E., Giroletti, M., Giovannini, G., et al. 2013, A\&A, 560, A23

Marchã, M. J. M., \& Caccianiga, A. 2013, MNRAS, 430, 2464

Marcha, M. J. M., Browne, I. W. A., Impey, C. D., \& Smith, P. S. 1996, MNRAS, 281,425

Massaro, E., Giommi, P., Leto, C., et al. 2009, A\&A, 495, 691

Massaro, F., D’Abrusco, R., Ajello, M., Grindlay, J. E., \& Smith, H. A. 2011, ApJ, 740, L48

Mauch, T., \& Sadler, E. M. 2007, MNRAS, 375, 931

Montero-Dorta, A. D., \& Prada, F. 2009, MNRAS, 399, 1106

Padovani, P., Perlman, E. S., Landt, H., Giommi, P., \& Perri, M. 2003, ApJ, 588, 128

Padovani, P., Giommi, P., Landt, H., \& Perlman, E. S. 2007, ApJ, 662, 182

Plotkin, R. M., Anderson, S. F., Brandt, W. N., et al. 2010, AJ, 139, 390

Polletta, M., Tajer, M., Maraschi, L., et al. 2007, ApJ, 663, 81

Raiteri, C. M., Villata, M., Carnerero, M. I., et al. 2014, MNRAS, 442, 629

Sadler, E. M., Ekers, R. D., Mahony, E. K., Mauch, T., \& Murphy, T. 2014, MNRAS, 438, 796

Sambruna, R. M., Maraschi, L., \& Urry, C. M. 1996, ApJ, 463, 444

Savolainen, T., Homan, D. C., Hovatta, T., et al. 2010, A\&A, 512, A24

Smith, A. G. 1996, in Blazar Continuum Variability, eds. H. R. Miller, J. R. Webb, \& J. C. Noble, ASP Conf. Ser., 110, 3

Stickel, M., Fried, J. W., Kuehr, H., Padovani, P., \& Urry, C. M. 1991, ApJ, 374, 431

Stocke, J. T., Morris, S. L., Gioia, I. M., et al. 1991, ApJS, 76, 813

Strauss, M. A., Weinberg, D. H., Lupton, R. H., et al. 2002, AJ, 124, 1810

Tremaine, S., Gebhardt, K., Bender, R., et al. 2002, ApJ, 574, 740

Tremonti, C. A., Heckman, T. M., Kauffmann, G., et al. 2004, ApJ, 613, 898

Urry, C. M., \& Padovani, P. 1991, ApJ, 371, 60

Urry, C. M., \& Padovani, P. 1995, PASP, 107, 803

Urry, C. M., \& Shafer, R. A. 1984, ApJ, 280, 569

Urry, C. M., Padovani, P., \& Stickel, M. 1991, ApJ, 382, 501

Wagner, S. J., Witzel, A., Heidt, J., et al. 1996, AJ, 111, 2187

Wolter, A., Caccianiga, A., della Ceca, R., \& Maccacaro, T. 1994, ApJ, 433, 29

Worthey, G., \& Ottaviani, D. L. 1997, ApJS, 111, 377

Wright, E. L., Eisenhardt, P. R. M., Mainzer, A. K., et al. 2010, AJ, 140, 1868

Zehavi, I., Blanton, M. R., Frieman, J. A., et al. 2002, ApJ, 571, 172 